\title{
Hadronic production of squark-squark pairs: the electroweak contributions
}

\author{
Jan Germer, ${ }^{a}$ Wolfgang Hollik, ${ }^{a}$ Edoardo Mirabella $^{b}$ and Maike K. Trenkel ${ }^{c}$ \\ a Max-Planck-Institut für Physik, \\ Föhringer Ring 6, D-80805 München, Germany \\ ${ }^{b}$ Institut de Physique Théorique, \\ CEA-Saclay, F-91191, Gif-sur-Yvette cedex, France \\ ${ }^{c}$ University of Wisconsin, \\ Madison, WI, 53706, U.S.A. \\ E-mail: germer@mppmu.mpg.de, hollik@mppmu.mpg.de, \\ edoardo.mirabella@cea.fr, trenkel@hep.wisc.edu
}

ABSTRACT: We compute the electroweak (EW) contributions to squark-squark pair production processes at the LHC within the framework of the Minimal Supersymmetric Standard Model (MSSM). Both tree-level EW contributions, of $\mathcal{O}\left(\alpha_{s} \alpha+\alpha^{2}\right)$, and next-to-leading order (NLO) EW corrections, of $\mathcal{O}\left(\alpha_{s}^{2} \alpha\right)$, are calculated. Depending on the flavor and chirality of the produced squarks, many interferences between EW-mediated and QCD-mediated diagrams give non-zero contributions at tree-level and NLO. We discuss the computational techniques and present an extensive numerical analysis for inclusive squark-squark production as well as for subsets and single processes. While the tree-level EW contributions to the integrated cross sections can reach the $20 \%$ level, the NLO EW corrections typically lower the LO prediction by a few percent.

Keywords: Supersymmetric Standard Model, NLO Computations, Hadronic Colliders 


\section{Contents}

1 Introduction 1

2 Classification of processes and tree-level cross sections 3

3 Virtual and real corrections of $\mathcal{O}\left(\alpha_{s}^{2} \alpha\right)$

3.1 Virtual corrections 6

3.2 Real corrections 8

3.2.1 Real photon emission $\quad 8$

$\begin{array}{llr}3.2 .2 & \text { Real gluon emission } & 9\end{array}$

$\begin{array}{ll}3.2 .3 & \text { Real quark emission }\end{array}$

$\begin{array}{lll}3.3 & \text { Factorization of initial-state collinear singularities } & 10\end{array}$

4 Numerical results $\quad 10$

4.1 Input Parameters 11

$\begin{array}{lll}4.2 & \text { Total hadronic cross sections } & 13\end{array}$

$\begin{array}{lll}4.3 & \text { Differential distributions } & 17\end{array}$

$\begin{array}{lll}5 & \text { Conclusions } & 21\end{array}$

$\begin{array}{ll}\text { A Tree-level cross sections } & 23\end{array}$

B Bremsstrahlung integrals $\quad 26$

B.1 Soft and collinear photon bremsstrahlung 26

$\begin{array}{lll}\text { B.2 Soft and collinear gluon bremsstrahlung } & 27\end{array}$

B.3 Collinear quark bremsstrahlung 28

C Hadronic cross sections for $\sqrt{S}=7 \mathrm{TeV} \quad 29$

$\begin{array}{ll}\text { D Feynman diagrams } & 29\end{array}$

\section{Introduction}

Supersymmetry (SUSY) [1] is one of the most appealing scenarios for physics beyond the Standard Model (SM). Being proposed as the only nontrivial extension to the space-time symmetry, it was found to have many nice phenomenological properties which overcome weaknesses of the SM. With SUSY particles at the TeV scale or below, the technical part of the hierarchy problem is solved by stabilizing the electroweak scale, in particular the mass of the Higgs boson. In addition, many supersymmetric extensions of the SM offer a dark matter candidate that gives the observed dark matter relict density [2]. Of particular 
interest is the Minimal Supersymmetric Standard Model (MSSM) [3-5] which provides a fit to electroweak precision observables and B-physics data with a $\chi^{2}$ comparable to the SM and a naturally light Higgs boson [6-8] and can explain the measured value of the anomalous magnetic moment $[9,10]$.

If supersymmetry is realized at the $\mathrm{TeV}$ scale, it will be probed at the Large Hadron Collider (LHC) at CERN. Indeed, the $95 \%$ C.L. area of the $\left(m_{0}, m_{1 / 2}\right)$ plane of the Constrained MSSM (CMSSM), lies largely within the region that can be explored within $1 \mathrm{fb}^{-1}$ of integrated luminosity [11]. Within the framework of the MSSM, imposing R-parity conservation, SUSY particles can only be produced in pairs. Among the potential SUSY discovery channels, the direct production of pairs of color-charged SUSY particles is of particular importance at hadron colliders since it proceeds via the strong interaction. Many searches for squarks and gluinos have thus already been performed at high-energy colliders. Results of the DØ and CDF collaboration can be found in e.g. [12]. Studies for the LHC are based on Monte Carlo simulations. It has been shown that there is the possibility of early SUSY discovery within $1 \mathrm{fb}^{-1}$ of data in the inclusive jets plus missing energy channel, provided that the SUSY particles are not too heavy [13].

First theoretical cross section predictions for squark and gluino pair production processes based on leading order (LO) calculations were made already many years ago [14-17]. Later calculations of next-to-leading order (NLO) in perturbative QCD [18, 19] could reduce theoretical uncertainties considerably and revealed corrections of typically $20-30 \%$. Recently also results beyond the one-loop level in QCD have become available [20-23], increasing the cross section by another $2-10 \%$, and stabilizing the prediction considerably.

For a reliable cross section prediction, also electroweak (EW) contributions have to be taken into account which are of the same size as the NNLO QCD contributions. The contributing processes are manifold and their interplay is nontrivial, in particular if not only QCD-mediated but also EW-mediated production channels exist at tree-level.

The latter arise from $q \bar{q}$ annihilation or $q q$ scattering, as well as photon-induced processes, and contribute at $\mathcal{O}\left(\alpha_{s} \alpha+\alpha^{2}\right)$. For squark-(anti)-squark production processes, the $q \bar{q}(q q)$-channels can rise the LO cross section by up to $20 \%[24,25]$. These contributions have also been studied in the context of non-minimal flavor violation [26] and explicit CP violation [27]. The photon-induced channels are typically more important for the pair production of lighter stops [28], and have a reduced impact on squark-anti-squark [29] and gluino-squark production [30].

NLO EW corrections contribute at $\mathcal{O}\left(\alpha_{s}^{2} \alpha\right)$ and have been investigated for stop-antistop [28, 31], squark-anti-squark [29], gluino-squark [30] and gluino-gluino [32] production processes. In this paper, we provide the yet-missing NLO EW corrections to squark-squark production. We give details on the NLO computation and present an elaborate numerical analysis of all squark-squark production processes, including the anti-particles.

In the context of all squark and gluino production processes, squark-squark production is of particular interest at the proton-proton collider LHC. The partonic process proceeds at LO from $q q$-induced diagrams only. Squark-anti-squark and gluino-gluino production require $q \bar{q}$ or $g g$ initial states instead. Since the final-state SUSY particles are very massive, an important contribution to the hadronic cross sections arises from the high- $x$ region where 
valence-quark densities dominate. As a result, squark-squark production has generally a higher tree-level yield than squark-anti-squark production and can be comparable to gluinogluino production depending on the precise squark-gluino mass configuration.

The outline of this paper is as follows: In section 2 we review the various tree-level contributions to squark-squark production and introduce some notation used throughout this paper. Section 3 shows the details to the NLO calculation of $\mathcal{O}\left(\alpha_{s}^{2} \alpha\right)$ and the strategy of the calculation. In section 4 we list the input parameters used in our numerical analysis and show hadronic cross sections and distributions for squark-squark production in protonproton collisions at the LHC with $\sqrt{S}=14 \mathrm{TeV}$. Different SUSY scenarios are considered and a scan over squark and gluino masses is performed. Analytic formulas for the tree-level cross section and expressions for the cross section in the soft and collinear singular region, numerical results for squark-squark production at the LHC with $\sqrt{S}=7 \mathrm{TeV}$, as well as the Feynman diagrams for the NLO calculation are collected in the appendix.

\section{Classification of processes and tree-level cross sections}

We consider the pair production of two squarks or two anti-squarks,

$$
P P \rightarrow \tilde{q}_{\alpha} \tilde{q}_{\beta}^{\prime}, \quad P P \rightarrow \tilde{q}_{\alpha}^{*} \tilde{q}_{\beta}^{* *}, \quad q, q^{\prime}=\{u, d, c, s\}
$$

where $\alpha, \beta=\{L, R\}$ label the chirality of the squarks, neglecting left-right mixing. At lowest order in QCD there is only one partonic channel for each process,

$$
\begin{aligned}
& q\left(p_{1}\right) q^{\prime}\left(p_{2}\right) \rightarrow \tilde{q}_{\alpha}\left(p_{3}\right) \tilde{q}_{\beta}^{\prime}\left(p_{4}\right), \\
& \bar{q}\left(p_{1}\right) \bar{q}^{\prime}\left(p_{2}\right) \rightarrow \tilde{q}_{\alpha}^{*}\left(p_{3}\right) \tilde{q}_{\beta}^{*}\left(p_{4}\right),
\end{aligned}
$$

where the initial-state quarks and the final-state squarks have to have the same flavor. We thus do not consider the production of top (bottom) squarks due to the vanishing (small) density of the corresponding quarks inside the proton. Moreover, b-squark production has specific different features and will be discussed separately. The unpolarized cross sections for squark-squark and anti-squark-anti-squark production are related by chargeconjugation. In the following we will refer to squark-squark production only, while the charge conjugated processes are properly taken into account in the numerical results.

Since the electroweak interaction is sensitive to flavor and chirality, one has to treat processes with final-state squarks of different chiralities or of different isospin separately, even in the limit of degenerate squark masses. CKM mixing effects are neglected in our discussion.

In total we distinguish 36 processes, resulting from the various combinations of squarks 


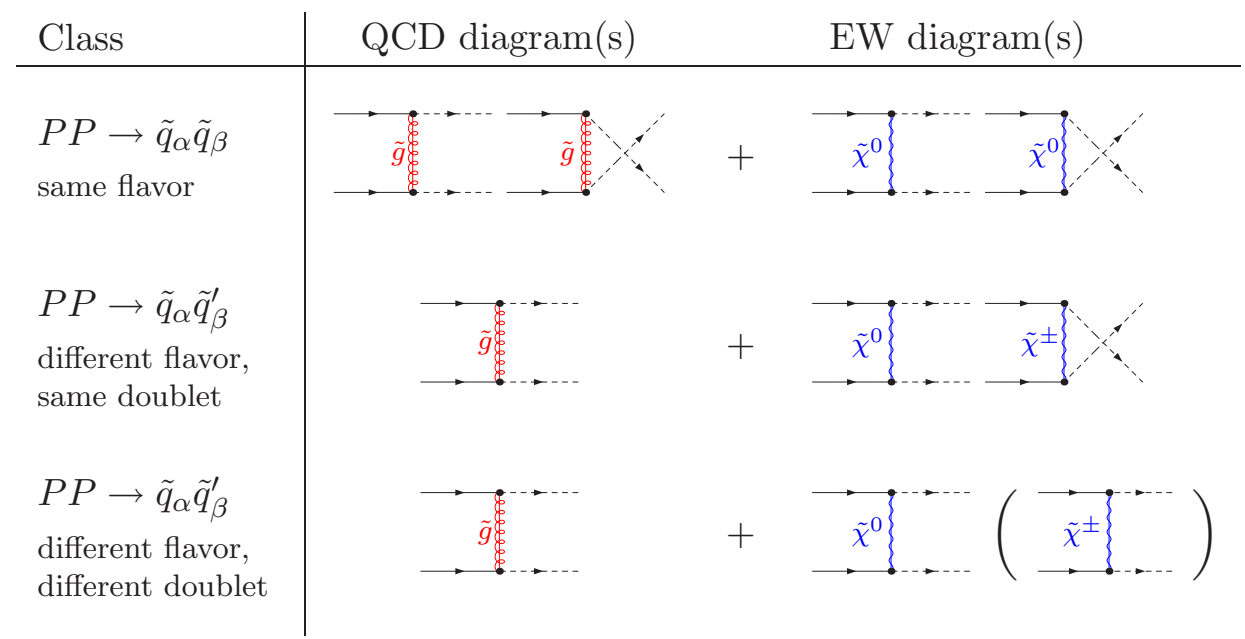

Figure 1. Parton-level Feynman diagrams for the three classes of squark-squark production at tree-level, where $\alpha, \beta=\{L, R\}$. The first class describes the production of two squarks of the same flavor, the second class that of two squarks of the same isospin doublet (but different flavor) and the third class refers to the production of two squarks belonging to different isospin doublets. In the third class, the subprocess in brackets cannot interfere with other diagrams due to different initial state particles. In all three classes, the final-state squarks are of the same generation as the initial-state quarks.

of different flavor or chirality in the final state. They can be classified as follows:

- production of two squarks of the same flavor,

$$
P P \rightarrow \tilde{u}_{\alpha} \tilde{u}_{\beta}, \tilde{d}_{\alpha} \tilde{d}_{\beta}, \tilde{c}_{\alpha} \tilde{c}_{\beta}, \tilde{s}_{\alpha} \tilde{s}_{\beta}, \quad\{\alpha \beta\}=\{L L, R R, L R\} .
$$

- production of two squarks belonging to the same $\mathrm{SU}(2)$ doublet,

$$
P P \rightarrow \tilde{u}_{\alpha} \tilde{d}_{\beta}, \tilde{c}_{\alpha} \tilde{s}_{\beta}, \quad\{\alpha \beta\}=\{L L, R R, L R, R L\} .
$$

- production of two squarks in different $\mathrm{SU}(2)$ doublets,

$$
P P \rightarrow \tilde{u}_{\alpha} \tilde{c}_{\beta}, \tilde{u}_{\alpha} \tilde{s}_{\beta}, \tilde{d}_{\alpha} \tilde{c}_{\beta}, \tilde{d}_{\alpha} \tilde{s}_{\beta}, \quad\{\alpha \beta\}=\{L L, R R, L R, R L\} .
$$

The corresponding tree-level diagrams of both QCD and EW origin are listed in figure 1. QCD diagrams are of $\mathcal{O}\left(\alpha_{s}\right)$, mediated by gluino exchange. EW diagrams are of $\mathcal{O}(\alpha)$ and mediated by neutralino or chargino exchange. Quarks and squarks are of the same flavor, also in the EW diagrams. The only exception is given by the two pure-EW charginomediated subprocesses $u d \rightarrow \tilde{d}_{L} \tilde{c}_{L}$ and $c d \rightarrow \tilde{u}_{L} \tilde{s}_{L}$ belonging to the third class, which contribute to $\tilde{d}_{L} \tilde{c}_{L}$ and $\tilde{u}_{L} \tilde{s}_{L}$ final states, respectively. Note that only $t$ - and $u$-channel diagrams are present, but no $s$-channel diagrams.

The appearance of both $t$ - and $u$-channel diagrams for chirality-diagonal $\tilde{q}_{\alpha} \tilde{q}_{\alpha}^{\prime}$ production gives rise to nonzero interferences between QCD and EW diagrams already at treelevel. ${ }^{1}$ The full tree-level contributions to the cross section are thus given by the $\mathcal{O}\left(\alpha_{s}^{2}\right)$ Born contribution and the $\mathcal{O}\left(\alpha_{s} \alpha+\alpha^{2}\right)$ EW contributions. Photon-induced squark-squark production is not possible at lowest order from charge and color conservation.

\footnotetext{
${ }^{1}$ In the non-diagonal case, $\tilde{q}_{L} \tilde{q}_{R}^{\prime}$ production, the interference contributions vanish as a consequence of the trivial squark mixing matrices in the limit of no L-R mixing, see also the discussion in appendix A.
} 
To keep track of the corresponding order in perturbation theory of the various contributions, we introduce the notation $d \hat{\sigma}^{a, b}\left[\mathcal{M}^{a, b}\right]$ in order to refer to the cross section [matrix element] at a given order $\mathcal{O}\left(\alpha_{s}^{a} \alpha^{b}\right)$ in the strong and electroweak couplings, respectively. Results are given in terms of the Mandelstam variables, defined as usual,

$$
\hat{s}=\left(p_{1}+p_{2}\right)^{2}, \quad \hat{t}=\left(p_{1}-p_{3}\right)^{2}, \quad \hat{u}=\left(p_{1}-p_{4}\right)^{2} .
$$

The differential partonic cross section for a given subprocess $q q^{\prime} \rightarrow \tilde{q}_{\alpha} \tilde{q}_{\beta}^{\prime}$ at LO can thus be written as

$$
d \hat{\sigma}^{2,0}(\hat{s})=\bar{\sum}\left|\mathcal{M}^{1,0}\right|^{2} \frac{d \hat{t}}{16 \pi \hat{s}^{2}}
$$

in terms of the squared lowest-order matrix element, $\mathcal{M}^{1,0}$, averaged (summed) over initial (final) state spin and color. Similarly, the pure EW differential cross section of $\mathcal{O}\left(\alpha^{2}\right)$ and the EW-QCD $\mathcal{O}\left(\alpha_{s} \alpha\right)$ interference contribution are given by

$$
\begin{aligned}
& d \hat{\sigma}^{0,2}(\hat{s})=\bar{\sum}\left|\mathcal{M}^{0,1}\right|^{2} \frac{d \hat{t}}{16 \pi \hat{s}^{2}}, \\
& d \hat{\sigma}^{1,1}(\hat{s})=\bar{\sum} 2 \operatorname{Re}\left\{\left(\mathcal{M}^{0,1}\right)^{*} \mathcal{M}^{1,0}\right\} \frac{d \hat{t}}{16 \pi \hat{s}^{2}},
\end{aligned}
$$

where $\mathcal{M}^{0,1}$ denotes the EW tree-level amplitude. Explicit expressions for the squared matrix elements are given in $[18,24]$. For completeness, we include a list of all tree-level differential cross sections in appendix A. ${ }^{2}$

The hadronic cross sections are obtained from the partonic cross sections by convolution with the respective parton luminosity function. At $\mathcal{O}\left(\alpha_{s}^{a} \alpha^{b}\right)$, it is given by

$$
\begin{aligned}
d \sigma^{a, b}(S) & =\int_{\tau_{0}}^{1} d \tau \frac{d L_{q q^{\prime}}}{d \tau} d \hat{\sigma}^{a, b}(\hat{s}), \\
\text { with } \quad \frac{d L_{q q^{\prime}}}{d \tau} & =\frac{1}{1+\delta_{q q^{\prime}}} \int_{\tau}^{1} \frac{d x}{x}\left[f_{q}^{A}\left(\frac{\tau}{x}, \mu_{F}\right) f_{q^{\prime}}^{B}\left(x, \mu_{F}\right)+f_{q}^{A}\left(x, \mu_{F}\right) f_{q^{\prime}}^{B}\left(\frac{\tau}{x}, \mu_{F}\right)\right]
\end{aligned}
$$

Here $\tau_{0}=\left(m_{\tilde{q}_{\alpha}}+m_{\tilde{q}_{\beta}^{\prime}}\right)^{2} / S$ is the production threshold, determined by the masses of the two squarks $m_{\tilde{q}_{\alpha}}$ and $m_{\tilde{q}_{\beta}^{\prime}}$. The parton distribution functions (PDFs) $f_{q}^{A}\left(x, \mu_{F}\right)$ give the probability to find a parton $q$ with momentum fraction $x$ inside hadron $A$ at a factorization scale $\mu_{F}$. At the LHC, both hadrons $A, B$ are protons $P . S$ and $\hat{s}=\tau S$ are the squared center-of-mass (c. m. ) energies of the hadronic and partonic processes, respectively.

\section{$3 \quad$ Virtual and real corrections of $\mathcal{O}\left(\alpha_{s}^{2} \alpha\right)$}

At $\mathcal{O}\left(\alpha_{s}^{2} \alpha\right)$, squark-squark production gets contributions from virtual corrections, real photon- and gluon emission, as well as real quark radiation. Ultraviolet (UV) as well as

\footnotetext{
${ }^{2}$ Note that we correct a wrong color factor of [24], which affects the pure EW $\mathcal{O}\left(\alpha^{2}\right)$ contribution. The numerical impact is negligible for all squark-squark production processes but it can be sizable in squarkanti-squark production channels where the $\mathcal{O}\left(\alpha_{s} \alpha\right)$ interference contribution is suppressed.
} 
(a)

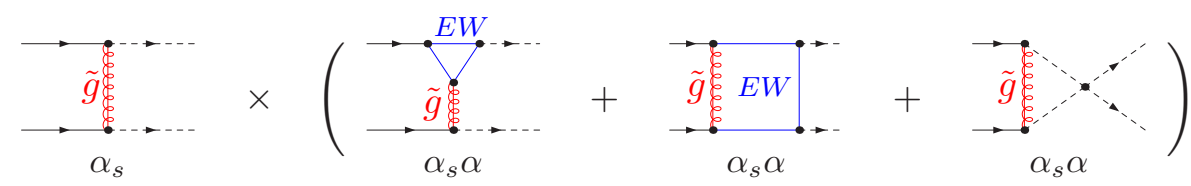

(b)
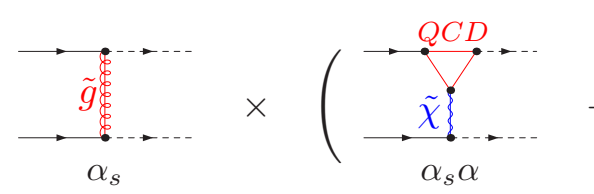

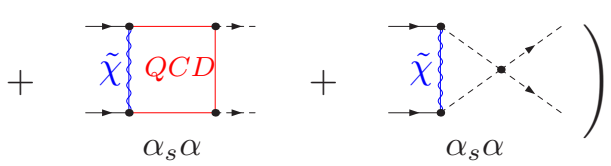

(c)
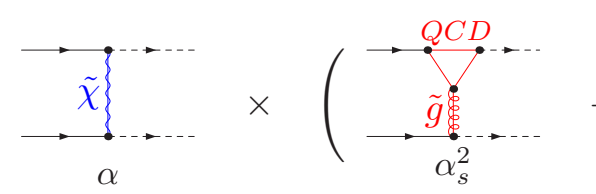

$+\frac{\overrightarrow{\tilde{g}} \xi_{\xi_{s}} \overrightarrow{{ }^{2}}}{\alpha_{s}^{2}}+$

$\left.+\underset{\alpha_{s}^{2}}{\rightarrow \vec{g}}\right)$

Figure 2. Sample of Feynman diagrams to illustrate the virtual contributions at $\mathcal{O}\left(\alpha_{s}^{2} \alpha\right)$. Three gauge invariant subsets of interferences occur at this order. The label of perturbative order is attached to each diagram. $E W$ refers to electroweakly interacting particles and $Q C D$ refers to strongly interacting particles in the loop insertions. The full sets of diagrams are shown in figures 13,14 , and 15 .

infrared (IR) and collinear singularities arise in the one-loop diagrams, see section 3.1. The IR singularities cancel in sufficiently inclusive observables once virtual and real photon and gluon bremsstrahlung corrections are added (see section 3.2). Remaining collinear singularities are universal and can be absorbed by redefining the PDFs, as described in section 3.3.

Diagrams and corresponding amplitudes are generated using FeynArts [33, 34]. The algebraic simplifications and numerical evaluation is done with help of FormCalc and LoopTools $[34,35]$. IR and collinear singularities are regularized by means of mass regularization, i.e. we introduce a fictitious mass for the photon and the gluon. Quarks are treated as massless, except where their masses are needed as regulators.

\subsection{Virtual corrections}

The virtual contributions are given by the interference of tree-level and one-loop diagrams. In practice three types of interferences occur at $\mathcal{O}\left(\alpha_{s}^{2} \alpha\right)$, as schematically depicted in figure 2. All three interference terms yield non-vanishing contributions to the cross section. For each subprocess, the partonic cross section can be written as

$$
\begin{aligned}
d \hat{\sigma}_{\text {virt. }}^{2,1}= & \frac{d \hat{t}}{16 \pi \hat{s}^{2}} \bar{\sum} 2 \operatorname{Re}\left\{\left(\mathcal{M}^{1,0}\right)^{*} \mathcal{M}_{(\mathrm{EW})}^{1,1}+\left(\mathcal{M}^{1,0}\right)^{*} \mathcal{M}_{(\mathrm{QCD})}^{1,1}\right\} \\
& +\frac{d \hat{t}}{16 \pi \hat{s}^{2}} \bar{\sum} 2 \operatorname{Re}\left\{\left(\mathcal{M}^{0,1}\right)^{*} \mathcal{M}^{2,0}\right\}
\end{aligned}
$$

The first line corresponds to $(a)$ and $(b)$ of figure 2 and is given by the interference of $\mathcal{M}^{1,0}$ with $\mathcal{M}^{1,1}$. The amplitude $\mathcal{M}^{1,1}$ is split into two parts, $\mathcal{M}_{(\mathrm{EW})}^{1,1}$ and $\mathcal{M}_{(\mathrm{QCD})}^{1,1}$, the first arising from tree-level QCD diagrams with EW insertions (figure 2a, right), and the latter from tree-level EW diagrams with QCD insertions (figure 2b, right). The second line in eq. (3.1), corresponding to figure 2c, is given by the interference of $\mathcal{M}^{0,1}$ with the 
pure-QCD one loop amplitude $\mathcal{M}^{2,0}$. Care has to be taken with diagrams containing a four-squark vertex. This vertex includes the electroweak as well as the strong coupling and the appropriate part has to be selected in each interference contribution to match the right order, as indicated in figure 2.

The full set of virtual corrections is UV finite after renormalization of the theory and the inclusion of the proper set of one-loop counterterms. The renormalization for squarksquark production proceeds in close analogy to that for squark-anti-squark production described in [29] and is sketched here only briefly. Each of the three interference subsets is gauge-independent by itself and can be renormalized separately.

In the first group, shown in figure 2a, UV singularities only arise from gluino-mediated amplitudes with weak insertions $\left(\mathcal{M}_{(\mathrm{EW})}^{1,1}\right)$. We include the diagrams with counterterms for the $q \tilde{g} \tilde{q}_{\alpha}$ vertex, see figure 13, and evaluate the renormalization constants at $\mathcal{O}(\alpha)$. At this order in the perturbative expansion we need to renormalize quark and squark fields, while the renormalization of gluino and strong coupling is not required. The regularization of the divergent amplitudes in this sector is done in dimensional reduction, and renormalization of quarks and squarks is performed in the on-shell scheme.

In the second case, figure $2 \mathrm{~b}$, neutralino- or chargino-mediated amplitudes with strong insertions $\left(\mathcal{M}_{(\mathrm{QCD})}^{1,1}\right)$ are considered. To obtain a UV-finite result, one needs to include diagrams containing counterterms for the $q \tilde{q} \tilde{\chi}^{0}$ vertex and, if arising, for the $q \tilde{q}^{\prime} \tilde{\chi}^{ \pm}$vertex, see figure 14. The renormalization constants have to be evaluated at $\mathcal{O}\left(\alpha_{s}\right)$ and no renormalization of the neutralino or chargino is required. Since the gluino does not enter this subset of one-loop amplitudes, it is thus sufficient to renormalize the quark and squark sector. As before, the divergent amplitudes are regularized in dimensional reduction and on-shell conditions are imposed to fix the (s)quark renormalization constants.

The third subset, figure 2c, refers to pure-QCD one-loop amplitudes, i.e. gluinomediated diagrams with strong insertions $\left(\mathcal{M}^{2,0}\right)$. In this case one has to renormalize the quark and squark sector as well as the gluino and the strong Yukawa coupling $\hat{g}_{s}$, which appears in the $q \tilde{q} \tilde{g}$ vertex. The renormalization constants in the corresponding amplitudes, see figure 15, have to be evaluated at $\mathcal{O}\left(\alpha_{s}\right)$. The strong scalar coupling $\hat{g}_{s}$ is related to the strong coupling $g_{s}$ via supersymmetry. To match the definition of the strong coupling constant used in the extraction of the PDFs, $g_{s}$ has to be given in the $\overline{\mathrm{MS}}$ scheme with the contributions from heavy particles subtracted in the running of $\alpha_{s}$. We thus regularize this part of the virtual corrections using dimensional regularization. Quarks and squarks are renormalized on-shell again, in the strong sector the $\overline{\mathrm{MS}}$ scheme is applied. Dimensional regularization however induces a finite difference between $g_{s}$ and $\hat{g}_{s}$ at the one-loop level and violates the supersymmetric relation between the two couplings [36]. We add the well-known finite shift in the definition of the renormalization constant for $\hat{g}_{s}$ in order to restore SUSY in the physical amplitudes [18].

All counterterms and renormalization constants are explicitly given in [29], appendix $\mathrm{B}$, and need not to be repeated here.

The virtual corrections also exhibit photonic and gluonic mass singularities of infrared (IR) and collinear origin. In $\mathcal{M}_{(\mathrm{EW})}^{1,1}$, mass singularities arise if two external particles 


$$
\begin{aligned}
& \text { photon bremsstrahlung } \\
& \left|\frac{\tilde{g} \frac{\xi}{\xi_{\xi}}}{\alpha_{s} \sqrt{\alpha}}\right|^{2} \\
& \text { gluon bremsstrahlung }
\end{aligned}
$$

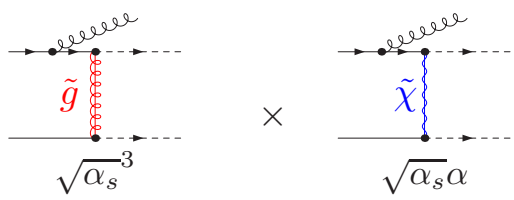

$$
\begin{aligned}
& \text { real quark radiation }
\end{aligned}
$$

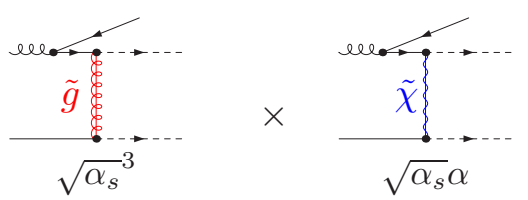

Figure 3. Sample of Feynman diagrams for the three subsets of real emission contributions at $\mathcal{O}\left(\alpha_{s}^{2} \alpha\right)$. The order in the perturbative expansion is specified for each diagram. The full sets of diagrams are given in the appendix, cf. figures 16, 17, and 18.

exchange a low-energetic massless photon while collinear singularities appear if one of the massless initial-state quarks splits collinearly into a quark and a photon. In order to obtain an IR finite result, real photon radiation at $\mathcal{O}\left(\alpha_{s}^{2} \alpha\right)$ has to be added. In contrast in $\mathcal{M}_{(\mathrm{QCD})}^{1,1}$, massless gluons running in the loops give rise to mass singularities in the soft and collinear limit. Similarly, the diagrams contributing to $\mathcal{M}^{2,0}$ suffer from gluonic IR and collinear singularities. Hence we have to include real gluon bremsstrahlung at $\mathcal{O}\left(\alpha_{s}^{2} \alpha\right)$ in order to cancel the IR singularities. We regularize the photonic singularities by means of mass regularization. Owing to the photon-like appearance of the gluon in the respective diagrams, it is also possible to regularize these IR singularities by a fictitious gluon mass.

\subsection{Real corrections}

Three independent bremsstrahlung processes contribute at $\mathcal{O}\left(\alpha_{s}^{2} \alpha\right)$, as depicted in figure 3 . Real photon and real gluon radiation processes have to be combined with the corresponding subset of virtual corrections to obtain an IR finite result. Also real quark radiation gives nonzero contributions from the interference of QCD and EW mediated diagrams and has to be included in the cross section at $\mathcal{O}\left(\alpha_{s}^{2} \alpha\right)$.

\subsubsection{Real photon emission}

The real photon emission at $\mathcal{O}\left(\alpha_{s}^{2} \alpha\right)$,

$$
q\left(p_{1}\right) q^{\prime}\left(p_{2}\right) \rightarrow \tilde{q}_{\alpha}\left(p_{3}\right) \tilde{q}_{\beta}^{\prime}\left(p_{4}\right) \gamma(k)
$$

is given by the squared matrix element of a QCD tree-level diagram with an external photon attached (see figure 3, top and figure 16 for the full set of diagrams). The integration over the photon phase space is IR divergent in the soft-photon region, i.e. for $k^{0} \rightarrow 0$. Further singularities arise in the collinear region if $p_{i} \cdot k \rightarrow 0$ for $i=\{1,2\}$. We use phase space slicing and apply a cut on the photon energy, $k^{0}>\delta_{s} \sqrt{\hat{s}} / 2$, and on the angle $\theta$ between 
the photon and incoming partons, $|\cos (\theta)|<1-\delta_{\theta}$, to split off the singular regions. In the hard, non-collinear region the integration is convergent and is performed numerically. The cross sections in the soft- and collinear region can be approximated analytically and are given in appendix B.1.

By combining the real photon emission with the virtual EW-type corrections (figure 2a), the soft singularities cancel. Remaining initial-state collinear singularities are universal and have to be absorbed via factorization in the PDFs, see section 3.3. In the following, we will refer to this UV-, IR- and collinear-finite combination as the EW-type corrections.

\subsubsection{Real gluon emission}

Real gluon bremsstrahlung at $\mathcal{O}\left(\alpha_{s}^{2} \alpha\right)$ proceeds via the partonic process

$$
q\left(p_{1}\right) q^{\prime}\left(p_{2}\right) \rightarrow \tilde{q}_{\alpha}\left(p_{3}\right) \tilde{q}_{\beta}^{\prime}\left(p_{3}\right) g(k) .
$$

It is given by the interference term of a QCD- and an EW tree-level diagram, both with an external gluon attached on (figure 3, center and figure 17). In the considered processes the gluon is Abelian like and we can treat soft and collinear singularities by mass regularization in close analogy to the photonic case. However the eikonal current has to be modified in order to take color correlations into account. Different to real photon emission, collinear singularities only arise for diagonal $\tilde{q}_{\alpha} \tilde{q}_{\alpha}, \tilde{u}_{L} \tilde{d}_{L}$, and $\tilde{c}_{L} \tilde{s}_{L}$ production. This can be seen by noticing that in the collinear cone the cross section becomes proportional to the corresponding $2 \rightarrow 2$ process, which in this case would be squark-squark production at $\mathcal{O}\left(\alpha_{s} \alpha\right)$; i.e. the interference of tree-level QCD and EW diagrams must be non-vanishing. Explicit expressions for the cross sections in the soft- and collinear regions are given in appendix B.2.

By combining real gluon emission and the two virtual QCD-type corrections (figure 2b,c), the IR singularities cancel. Remaining collinear singularities are again absorbed into the PDFs, as described in section 3.3. In the following we will refer to this UV-, IRand collinear-finite combination as the QCD-type corrections.

\subsubsection{Real quark emission}

Finally, also real quark radiation contributes at $\mathcal{O}\left(\alpha_{s}^{2} \alpha\right)$,

$$
\begin{aligned}
g\left(p_{1}\right) q\left(p_{2}\right) & \rightarrow \tilde{q}_{\alpha}\left(p_{3}\right) \tilde{q}_{\beta}^{\prime}\left(p_{4}\right) \bar{q}^{\prime}(k), \\
\text { and if } q \neq q^{\prime} \quad g\left(p_{1}\right) q^{\prime}\left(p_{2}\right) & \rightarrow \tilde{q}_{\alpha}\left(p_{3}\right) \tilde{q}_{\beta}^{\prime}\left(p_{4}\right) \bar{q}(k),
\end{aligned}
$$

via the interference of a QCD-type diagram with an EW-type diagram, as shown in figure 3, bottom (see figure 18 for the complete listing of diagrams). This process can be regarded as completely independent to the virtual corrections, since it is IR finite by itself. However it has to be taken into account in a consistent analysis of electroweak corrections up to $\mathcal{O}\left(\alpha_{s}^{2} \alpha\right)$. Initial-state collinear singularities are present for processes with non-vanishing tree-level interferences. The cross section in the collinear region is given in appendix B.3. As before, these singularities are absorbed via factorization into the PDFs, see section 3.3. 
Different to photon and gluon bremsstrahlung, the internal gluino, neutralino or chargino can go on-shell in specific SUSY scenarios, if heavier than one of the external squarks. In these cases, we include a Breit-Wigner width for the resonant particle in the corresponding propagators to regularize the poles. Note that physical resonances do not occur. This is different to the case of real quark radiation in e.g. gluino-squark production processes [30], where internal squarks can go on-shell in both the EW- and the QCD-mediated diagrams.

\subsection{Factorization of initial-state collinear singularities}

The remaining collinear singularities have to be absorbed by redefining the PDFs. At $\mathcal{O}\left(\alpha_{s}^{2} \alpha\right)$ this can be achieved by the replacement $[37,38]$

$$
\begin{aligned}
f_{q}\left(x, \mu_{F}\right) \rightarrow & f_{q}\left(x, \mu_{F}\right)\left(1-\frac{\alpha e_{q}^{2}+\alpha_{s} C_{F}}{\pi} \kappa_{v+s}-\frac{1}{4} \frac{\alpha e_{q}^{2}}{\pi} f_{v+s}\right) \\
& -\int_{x}^{1-\delta_{s}} \frac{d z}{z} f_{q}\left(\frac{x}{z}, \mu_{F}\right)\left(\frac{\alpha e_{q}^{2}+\alpha_{s} C_{F}}{2 \pi} \kappa_{c}(z)-\frac{\alpha e_{q}^{2}}{2 \pi} f_{c}(z)\right) \\
& -\int_{x}^{1} \frac{d z}{z} f_{g}\left(\frac{x}{z}, \mu_{F}\right) \frac{\alpha_{s} C_{F}}{2 \pi} P_{q g}(z) \ln \left(\frac{\mu_{F}^{2}}{m_{q}^{2}}\right),
\end{aligned}
$$

where $e_{q}$ denotes the electric charge of quark $q, C_{F}=4 / 3$, and

$$
\begin{aligned}
& \kappa_{v+s}=1-\ln \delta_{s}-\ln ^{2} \delta_{s}+\left(\ln \delta_{s}+\frac{3}{4}\right) \ln \left(\frac{\mu_{F}^{2}}{m_{q}^{2}}\right), \\
& \kappa_{c}(z)=P_{q q}(z) \ln \left(\frac{\mu_{F}^{2}}{m_{q}^{2}} \frac{1}{(1-z)^{2}}-1\right) .
\end{aligned}
$$

The factorization-scheme dependent functions are

$$
\begin{aligned}
& f_{v+s}=9+\frac{2 \pi^{2}}{3}+3 \ln \delta_{s}-2 \ln ^{2} \delta_{s} \\
& f_{c}(z)=P_{q q}(z) \ln \left(\frac{1-z}{z}\right)-\frac{3}{2} \frac{1}{1-z}+2 z+3,
\end{aligned}
$$

with the splitting functions

$$
P_{q q}(z)=\frac{1+z^{2}}{1-z}, \quad P_{q g}(z)=z^{2}+\left(1-z^{2}\right) .
$$

The factorization is done in the $\overline{\mathrm{MS}}$ scheme at NLO QCD and in the physical DIS scheme at NLO EW. The replacement of the PDFs in eq. (2.7) gives further contributions of $\mathcal{O}\left(\alpha_{s}^{2} \alpha\right)$ to the total cross section. The first and second line in eq. (3.5) cancel the remaining singularities in the EW-type and QCD-type corrections. The third line in eq. (3.5) cancels the collinear singularities in the real quark radiation.

\section{Numerical results}

In the following we illustrate the impact of the EW contributions on the production cross section. Since we have 36 processes contributing to squark-squark production and the 
same amount for anti-squark-anti-squark production, we present (at least partly) inclusive results. We refer to four different combinations of (anti-)squarks in the final state, which differ with respect to the chirality of the produced particles:

- $\tilde{q}_{L} \tilde{q}_{L}^{\prime}$ or "LL" refers to the inclusive production of two left-handed squarks and two left-handed anti-squarks.

- $\tilde{q}_{L} \tilde{q}_{R}^{\prime}$ or "LR" refers to the inclusive production of one left-handed and one righthanded squark and the charge conjugated process.

- $\tilde{q}_{R} \tilde{q}_{R}^{\prime}$ or "RR" refers to the inclusive production of two right-handed squarks and two right-handed anti-squarks.

- $\tilde{q} \tilde{q}^{\prime}$ or "incl." refers to the inclusive production of all (anti-)squarks. It is given by the sum of the three cases above, taking all 72 subprocesses of squark-squark and anti-squark-anti-squark final states into account.

We focus here on these chirality-based classes since squarks of different chiralities are, in principle, experimentally distinguishable by their decay chains, see e.g. section 5.1.2 of [39].

In the discussion we refer to the following quantities, based on the cross section definitions in section 2. The leading order cross section is denoted by $\sigma^{\text {Born }}=\sigma^{2,0}$. The tree-level EW and the NLO EW contributions to the cross section are labeled by

$$
\Delta \sigma^{\text {tree } \mathrm{EW}}=\left(\sigma^{1,1}+\sigma^{0,2}\right), \quad \Delta \sigma^{\mathrm{NLO} \mathrm{EW}}=\sigma^{2,1},
$$

respectively, and $\Delta \sigma^{\mathrm{EW}}=\Delta \sigma^{\text {tree } \mathrm{EW}}+\Delta \sigma^{\mathrm{NLO}} \mathrm{EW}$ will be referred to as the EW contribution. The total sum of the LO cross section with the EW contributions is denoted by $\sigma^{\mathrm{NLO}}=\sigma^{\mathrm{Born}}+\Delta \sigma^{\mathrm{EW}}$. Relative EW contributions are defined by

$\delta^{\text {tree } \mathrm{EW}}=\Delta \sigma^{\text {tree } \mathrm{EW}} / \sigma^{\text {Born }}, \quad \delta^{\mathrm{NLO} \mathrm{EW}}=\Delta \sigma^{\mathrm{NLO} \mathrm{EW}} / \sigma^{\mathrm{Born}}, \quad \delta^{\mathrm{EW}}=\Delta \sigma^{\mathrm{EW}} / \sigma^{\text {Born }}$.

In distributions $\delta$ denotes the relative $\mathrm{EW}$ contribution defined as $\delta=\left(\mathcal{O}_{\mathrm{NLO}}-\right.$ $\left.\mathcal{O}_{\text {Born }}\right) / \mathcal{O}_{\text {Born }}$, where $\mathcal{O}$ is a generic observable and $\mathcal{O}_{\text {NLO }}$ is the sum of the Born and the EW contribution.

\subsection{Input Parameters}

The Standard Model input parameters are chosen in correspondence with [40, 41],

$$
\begin{aligned}
M_{Z} & =91.1876 \mathrm{GeV}, & M_{W} & =80.4247685 \mathrm{GeV}, \\
\alpha^{-1} & =137.036, & \alpha_{s}\left(M_{Z}\right) & =0.119, \\
m_{t} & =170.9 \mathrm{GeV}, & m_{b}^{\text {OS }} & =4.7 \mathrm{GeV} .
\end{aligned}
$$

The strong coupling constant $\alpha_{s}$ has been defined in the $\overline{\mathrm{MS}}$ scheme using the two-loop renormalization group equation with five light flavors.

For the SUSY parameters, we refer to three benchmark mSUGRA scenarios, the SPS1a' scenario, the SPS2, and SPS5 scenario [40, 42]. The SPS1a' scenario can be considered as 


\begin{tabular}{|c|c|c|c|c|c|}
\hline & $\boldsymbol{m}_{\mathbf{0}}$ & $\boldsymbol{m}_{\mathbf{1} \boldsymbol{\mathbf { 2 }}}$ & $\boldsymbol{A}_{\mathbf{0}}$ & $\tan \boldsymbol{\beta}$ & $\operatorname{sign}(\boldsymbol{\mu})$ \\
\hline SPS1a $^{\prime}$ & $70 \mathrm{GeV}$ & $250 \mathrm{GeV}$ & $-300 \mathrm{GeV}$ & 10 & + \\
\hline SPS2 & $1450 \mathrm{GeV}$ & $300 \mathrm{GeV}$ & 0 & 9.66 & + \\
\hline SPS5 & $150 \mathrm{GeV}$ & $300 \mathrm{GeV}$ & $-1000 \mathrm{GeV}$ & 4.82 & + \\
\hline
\end{tabular}

Table 1. High energy input parameters for the different SUSY scenarios considered. The mass parameters $m_{0}, m_{1 / 2}$ and $A_{0}$ are given at the GUT scale, $\tan \beta$ is evaluated at $M_{\mathrm{SUSY}}=1 \mathrm{TeV}$.

\begin{tabular}{|c|c|c|c|c|c|c|c|}
\hline & $\tilde{\boldsymbol{u}}_{\boldsymbol{L}}$ & $\tilde{\boldsymbol{u}}_{\boldsymbol{R}}$ & $\tilde{\boldsymbol{d}}_{\boldsymbol{L}}$ & $\tilde{\boldsymbol{d}}_{\boldsymbol{R}}$ & $\tilde{\boldsymbol{g}}$ & $\tilde{\boldsymbol{\chi}}_{\mathbf{1}}^{0}$ & $\tilde{\boldsymbol{\chi}}_{\mathbf{1}}^{ \pm}$ \\
\hline SPS1a $^{\prime}$ & 561 & 543 & 566 & 539 & 609 & 101 & 180 \\
\hline SPS2 & 1559 & 1554 & 1561 & 1555 & 785 & 120 & 199 \\
\hline SPS5 & 677 & 655 & 681 & 654 & 724 & 123 & 225 \\
\hline
\end{tabular}

Table 2. On-shell masses of the squarks, the gluino, and the lightest neutralino and chargino within the different SUSY scenarios considered. All masses are given in GeV.

a "typical" mSUGRA scenario. It has been proposed by the SPA convention and should be used for comparisons with other calculations. The SPS2 scenario features relatively heavy squarks with light charginos and neutralinos and a gluino lighter than the squarks. The SPS5 scenario leads to a very light $\tilde{t}_{1}$ with moderate light-flavor squark masses. In each scenario, the particle spectrum is determined by universal GUT scale parameters, cf. table 1, which act as boundary conditions for the renormalization group running of the soft-breaking parameters. We use the program Softsusy [43] to evolve the soft-breaking parameters down to the SUSY scale $M_{\mathrm{SUSY}}$. We choose a common SUSY scale $M_{\mathrm{SUSY}}=1 \mathrm{TeV}$ for all scenarios, in reference to the SPA convention. At $M_{\mathrm{SUSY}}$ a consistent translation of the squark masses into the on-shell scheme is performed. The left-handed down-type squark is treated as a dependent mass parameter, fixed by $\mathrm{SU}(2)$ invariance. It is set to its corresponding on-shell value obtained at one-loop accuracy according to [44]. The on-shell mass parameters for the light flavor squarks together with the masses of the gluino and the lightest neutralino/chargino are summarized in table 2.

The technical cuts needed for the regularization of soft and collinear singularities are set to $\delta_{s}=10^{-3} \sqrt{\hat{s}}$ and $\delta_{\theta}=10^{-4}$. We checked numerically that these values are sufficiently small to justify the eikonal approximation. The results presented in this section are computed using the MRST2004QED parton distribution functions [45] and setting the hadronic center of mass energy to $\sqrt{S}=14 \mathrm{TeV}$. For comparison, we give results at $\sqrt{S}=7 \mathrm{TeV}$ in appendix $\mathrm{C}$.

Figure 4 shows the scale dependence for the SPS1a' scenario. Here we are inclusive with respect to processes that contribute at $\mathcal{O}\left(\alpha_{s} \alpha\right)$. We compare the tree-level EW contribution with the cross section including the EW NLO contributions at $\mathcal{O}\left(\alpha_{s}^{2} \alpha\right)$, i.e. one order higher in the strong coupling. In the left panel, factorization and renormalization scales are identified, $\mu=\mu_{R}=\mu_{F}=\bar{m}_{\tilde{q}}$ with $\bar{m}_{\tilde{q}}$ being the average mass of the light-flavor squarks. In the right panel the renormalization scale is fixed and only the factorization scale is varied. One finds that the scale dependence of the hadronic cross section is considerably reduced 


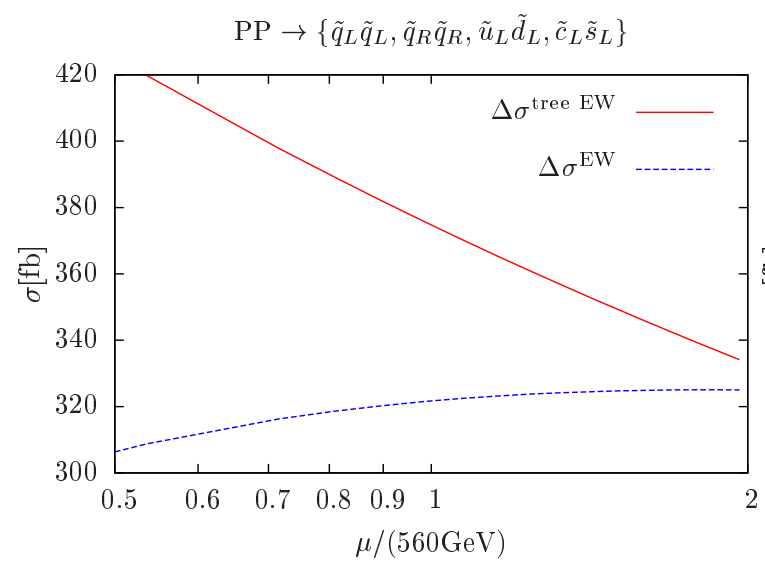

(a)

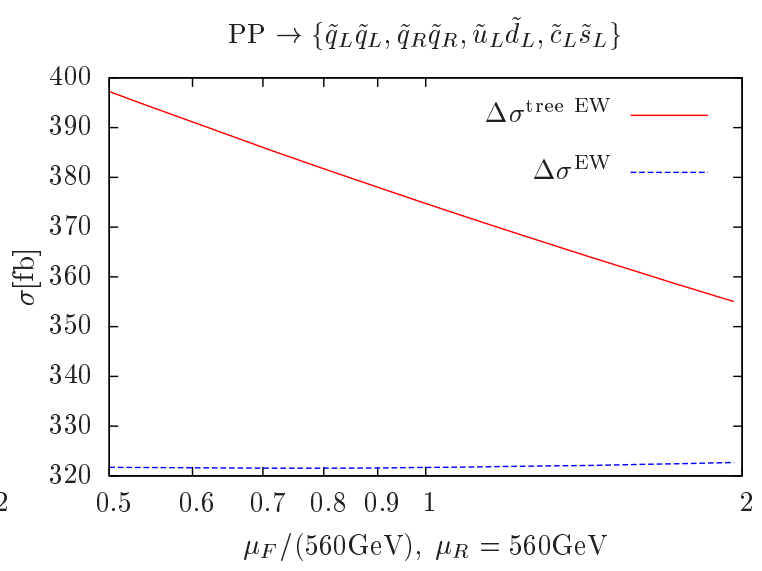

(b)

Figure 4. Dependence of the hadronic cross section $\sigma$ on the renormalization and factorization scale $\mu_{R}$ and $\mu_{F}$ for the SPS1a' scenario: (a) both scales are set to a common value $\mu_{R}=\mu_{F}=\mu$ with $\mu$ varied by a factor two around $\bar{m}_{\tilde{q}}=560 \mathrm{GeV}$, (b) variation of $\mu_{F}$, with fixed $\mu_{R}=\bar{m}_{\tilde{q}}$. Only processes that contribute at $\mathcal{O}\left(\alpha_{s} \alpha\right)$ are considered. The red line shows the EW tree-level contribution to the cross section, while the blue curve shows the EW contribution up to NLO EW.

\begin{tabular}{|c|c|c|c||c|c|c|}
\hline $\mathbf{S P S 1 a}^{\prime}$ & $\begin{array}{c}\boldsymbol{\sigma}^{\text {Born }} \\
\mathcal{O}\left(\alpha_{s}^{2}\right)\end{array}$ & $\begin{array}{c}\Delta \boldsymbol{\sigma}^{\text {tree EW }} \\
\mathcal{O}\left(\alpha_{s} \alpha+\alpha^{2}\right)\end{array}$ & $\begin{array}{c}\boldsymbol{\Delta} \boldsymbol{\sigma}^{\text {NLO EW }} \\
\mathcal{O}\left(\alpha_{s}^{2} \alpha\right)\end{array}$ & $\boldsymbol{\delta}^{\text {tree EW }}$ & $\boldsymbol{\delta}^{\text {NLO EW }}$ & $\boldsymbol{\delta}^{\text {EW }}$ \\
\hline$\tilde{\boldsymbol{q}}_{\boldsymbol{L}} \tilde{\boldsymbol{q}}_{\boldsymbol{L}}^{\prime}$ & $1717.6(8)$ & $378.9(1)$ & $-74.8(6)$ & $22.1 \%$ & $-4.4 \%$ & $17.7 \%$ \\
\hline$\tilde{\boldsymbol{q}}_{\boldsymbol{R}} \tilde{\boldsymbol{q}}_{\boldsymbol{R}}^{\prime}$ & $1981.9(7)$ & $31.81(2)$ & $-1.60(9)$ & $1.6 \%$ & $-0.1 \%$ & $1.5 \%$ \\
\hline$\tilde{\boldsymbol{q}}_{\boldsymbol{L}} \tilde{\boldsymbol{q}}_{\boldsymbol{R}}^{\prime}$ & $1743.8(4)$ & $2.538(1)$ & $-70.71(4)$ & $0.1 \%$ & $-4.1 \%$ & $-3.9 \%$ \\
\hline \hline$\tilde{\boldsymbol{q}} \tilde{\boldsymbol{q}}^{\prime}$ & $5443(1)$ & $413.3(1)$ & $-147.1(6)$ & $7.6 \%$ & $-2.7 \%$ & $4.9 \%$ \\
\hline
\end{tabular}

Table 3. Hadronic cross sections in femtobarn (fb) for squark-squark production at the LHC within the SPS1a' scenario for $\sqrt{S}=14 \mathrm{TeV}$. Shown are the LO cross section, the tree-level EW as well as NLO EW contributions and the relative corrections as defined in the text. Anti-particles are included. The numbers in brackets refer to the integration uncertainty in the last digit.

by taking the EW NLO contributions into account. The residual uncertainty arises mostly from the choice of the renormalization scale while the dependence on the factorization scale variation is mild at NLO. It is interesting to note that the impact of QED effects in the evolution of the PDFs is small. This feature, already pointed out in [46], has been explicitly checked using the NLO QCD PDF provided by the MRST 2004 NNLO set [47], which does not include QED effects. In the following, the factorization and renormalization scales are set to the common value $\mu=\mu_{R}=\mu_{F}=\bar{m}_{\tilde{q}}$.

\subsection{Total hadronic cross sections}

Tables 3-5 give the results for the hadronic cross sections for squark-squark production at the LHC at $\sqrt{S}=14 \mathrm{TeV}$ within the SPS1a', SPS2, and SPS5 scenario, respectively. We refer to the production of squarks of different chiralities separately. Renormalization and factorization scales are set to $\mu=560 \mathrm{GeV}$ (SPS1a'), $\mu=1560 \mathrm{GeV}$ (SPS2) and 


\begin{tabular}{|c|c|c|c||c|c|c|}
\hline SPS2 & $\begin{array}{c}\boldsymbol{\sigma}^{\text {Born }} \\
\mathcal{O}\left(\alpha_{s}^{2}\right)\end{array}$ & $\begin{array}{c}\boldsymbol{\Delta} \boldsymbol{\sigma}^{\text {tree EW }} \\
\mathcal{O}\left(\alpha_{s} \alpha+\alpha^{2}\right)\end{array}$ & $\begin{array}{c}\boldsymbol{\Delta} \boldsymbol{\sigma}^{\text {NLO EW }} \\
\mathcal{O}\left(\alpha_{s}^{2} \alpha\right)\end{array}$ & $\boldsymbol{\delta}^{\text {tree EW }}$ & $\boldsymbol{\delta}^{\text {NLO EW }}$ & $\boldsymbol{\delta}^{\text {EW }}$ \\
\hline$\tilde{\boldsymbol{q}}_{\boldsymbol{L}} \tilde{\boldsymbol{q}}_{\boldsymbol{L}}^{\prime}$ & $7.359(1)$ & $1.0326(2)$ & $-0.5776(7)$ & $14.0 \%$ & $-7.8 \%$ & $6.2 \%$ \\
\hline$\tilde{\boldsymbol{q}}_{\boldsymbol{R}} \tilde{\boldsymbol{q}}_{\boldsymbol{R}}^{\prime}$ & $7.529(1)$ & $0.1005(1)$ & $-0.0052(1)$ & $1.3 \%$ & $-0.1 \%$ & $1.3 \%$ \\
\hline$\tilde{\boldsymbol{q}}_{\boldsymbol{L}} \tilde{\boldsymbol{q}}_{\boldsymbol{R}}^{\prime}$ & $14.651(1)$ & $0.0136(1)$ & $-0.8676(2)$ & $0.1 \%$ & $-5.9 \%$ & $-5.8 \%$ \\
\hline \hline$\tilde{\boldsymbol{q}} \tilde{\boldsymbol{q}}^{\prime}$ & $29.539(2)$ & $1.1468(2)$ & $-1.4506(7)$ & $3.9 \%$ & $-4.9 \%$ & $-1.0 \%$ \\
\hline
\end{tabular}

Table 4. Same as table 3 but for the SPS2 scenario.

\begin{tabular}{|c|c|c|c||c|c|c|}
\hline SPS5 & $\begin{array}{c}\boldsymbol{\sigma}^{\text {Born }} \\
\mathcal{O}\left(\alpha_{s}^{2}\right)\end{array}$ & $\begin{array}{c}\boldsymbol{\Delta} \boldsymbol{\sigma}^{\text {tree EW }} \\
\mathcal{O}\left(\alpha_{s} \alpha+\alpha^{2}\right)\end{array}$ & $\begin{array}{c}\boldsymbol{\Delta} \boldsymbol{\sigma}^{\text {NLO EW }} \\
\mathcal{O}\left(\alpha_{s}^{2} \alpha\right)\end{array}$ & $\boldsymbol{\delta}^{\text {tree EW }}$ & $\boldsymbol{\delta}^{\text {NLO EW }}$ & $\boldsymbol{\delta}^{\text {EW }}$ \\
\hline$\tilde{\boldsymbol{q}}_{\boldsymbol{L}} \tilde{\boldsymbol{q}}_{\boldsymbol{L}}^{\prime}$ & $774.7(1)$ & $185.71(4)$ & $-35.9(1)$ & $24.0 \%$ & $-4.6 \%$ & $19.3 \%$ \\
\hline$\tilde{\boldsymbol{q}}_{\boldsymbol{R}} \tilde{\boldsymbol{q}}_{\boldsymbol{R}}^{\prime}$ & $888.0(1)$ & $16.332(5)$ & $-0.69(2)$ & $1.8 \%$ & $-0.1 \%$ & $1.8 \%$ \\
\hline$\tilde{\boldsymbol{q}}_{\boldsymbol{L}} \tilde{\boldsymbol{q}}_{\boldsymbol{R}}^{\prime}$ & $758.00(9)$ & $1.1559(3)$ & $-33.68(1)$ & $0.2 \%$ & $-4.4 \%$ & $-4.3 \%$ \\
\hline \hline$\tilde{\boldsymbol{q}} \tilde{\boldsymbol{q}}^{\prime}$ & $2420.7(3)$ & $203.20(4)$ & $-70.3(1)$ & $8.4 \%$ & $-2.9 \%$ & $5.5 \%$ \\
\hline
\end{tabular}

Table 5. Same as table 3 but for the SPS5 scenario.

$\mu=666 \mathrm{GeV}$ (SPS5). We checked that the results for the tree-level EW contributions numerically agree with those quoted for the SPS1a scenario in [24]. Differences between [24] and tables $3-5$ are related to the choice of input parameters and to the strong factorization scale dependence of the tree-level EW contributions, see figure 4.

The Born cross section is QCD mediated and does not depend on the chirality of the produced squarks. Indeed the cross sections for the diagonal production of two squarks, LL or RR, become equal for degenerate masses. The Born cross section for non-diagonal LR production, however, is different in general since the final-state particles are distinguishable. In the SPS1a' and SPS5 scenario it happens to be of similar size as the LL and RR production cross sections. In the SPS2 scenario, however, the LR cross section is enhanced and accounts for $50 \%$ of the total cross section for inclusive squark-squark production. As we will see below, cf. figure 7 (b), the relative yield of the LR production cross section is determined by the ratio of squark and gluino masses, becoming more important if the exchanged gluino is lighter than the final-state squarks.

Due to the nature of the electroweak interaction, the EW cross section contributions depend strongly on the chirality of the produced squarks. The tree-leel EW contributions have been studied extensively in [24]. Their impact is largest in case of the LL production of two $\mathrm{SU}(2)$ gauged left-handed squarks $(15-25 \%$ in the considered scenarios). For RR production, where only $\mathrm{U}(1)_{Y}$ couplings enter the EW-mediated diagrams, the treelevel EW contributions are around one order of magnitude smaller. They are even further suppressed for LR production, where the $\mathcal{O}\left(\alpha_{s} \alpha\right)$ tree-level interference contributions are completely absent. Also, LR production is induced by initial-state quarks of opposite helicities and thus suffers additionnally from a $p$ wave suppression. For a more detailed discussion we refer the reader to [24]. 

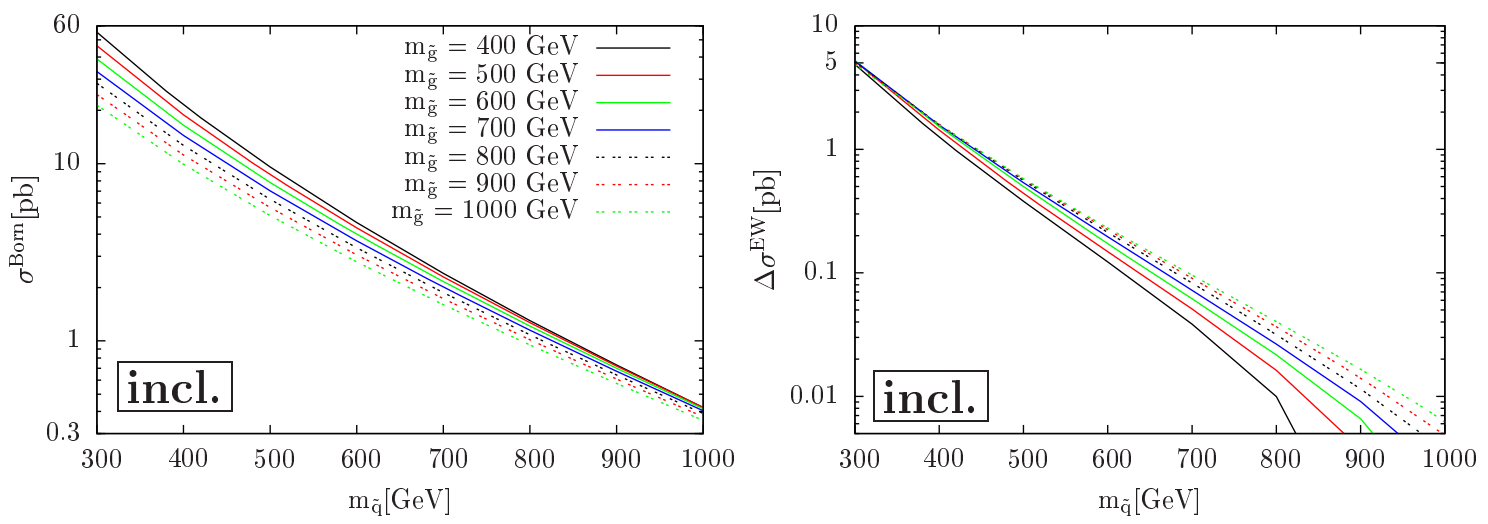

Figure 5. Hadronic Born cross section (left) and EW contributions (right) for inclusive squarksquark production as a function of a common squark mass $m_{\tilde{q}}$. Different gluino masses $m_{\tilde{g}}$ are considered, all other parameters are set to their SPS1a ${ }^{\prime}$ values.

The situation is different for the NLO EW contributions. These are equally important in the case of LL and LR production (reducing the LO prediction by about $4-8 \%$ in the considered scenarios), but negligible for RR production. In all three cases the NLO EW corrections are negative and partially compensate the EW tree-level contributions. Summing over all processes, the EW contributions to the total cross section for inclusive squark-squark production decrease from about $8 \%$ to about $5 \%$ in SPS1a ${ }^{\prime}$ and SPS5 after the inclusion of NLO EW corrections. In the SPS2 scenario, where LR production is the dominant production mechanism, the NLO EW corrections even overcompensate the EW tree-level contributions and the result turns negative.

In order to further investigate the dependence of the EW contributions on squark and gluino masses, we perform a parameter scan on those quantities. The independent squark masses are chosen equal to a common value $m_{\tilde{q}}$, with the dependent fourth squark mass set to its corresponding on-shell value. All other parameters are fixed to their SPS1a' values. The renormalization and the factorization scale are set to $\mu_{R}=\mu_{F}=m_{\tilde{q}}$.

To start with, we show in figure 5 the Born cross section (left panel) and the EW cross section contributions (right panel) for inclusive squark-squark production as a function of the common squark mass and for different values of the gluino mass. Both the Born and the EW contribution strongly decrease for growing squark masses. While the Born cross section is quite sensitive to the gluino mass for low squark masses, the EW contribution is almost independent in this regime. For high squark masses the behavior is vice versa.

Figure 6 shows the $K$ factor, defined as $K=\sigma^{\mathrm{NLO}} / \sigma^{\text {Born }}$, for the same parameter range as considered in figure 5 . The three different combinations of chiralities of the finalstate squarks, as well as the inclusive case are considered separately. The $K$ factor is largest for two left-handed squarks in the final state. Here, the EW contributions alter the LO cross section prediction between $10-50 \%$, being most important in case of light squarks and a heavy gluino. The EW contribution is enhanced by the large tree-level EW contribution. In the case of RR production, the EW contributions are below $3 \%$ in most parts of the parameter space. For LR production the EW contributions are mainly given by 

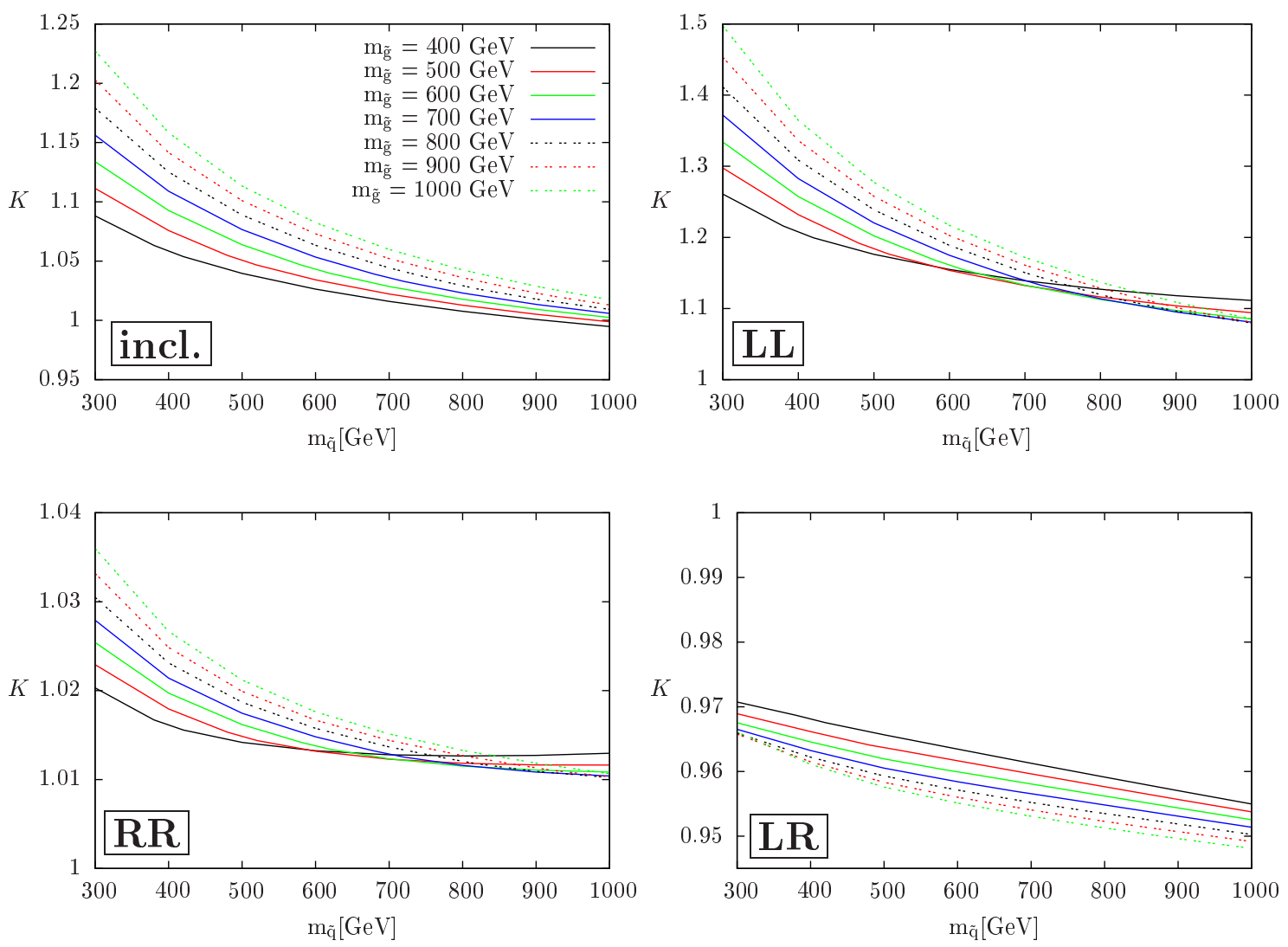

Figure 6. $K$ factor defined as $K=\sigma^{\mathrm{NLO}} / \sigma^{\text {Born }}$, as a function of a common squark mass. Different gluino masses $m_{\tilde{g}}$ are considered, all other parameters are set to their SPS1a' values. The labels "incl." and "LL", "RR", "LR" refer to inclusive squark-squark production and chirality-grouped subprocesses as explained in the text.

the NLO EW corrections, leading to a $K$ factor smaller than unity. The LO cross section is reduced by $-3 \%$ to $-5 \%$, most strongly in a scenario with heavy squarks and a heavy gluino. Altogether one finds for inclusive squark-squark production EW contributions that range from $9 \%$ for $m_{\tilde{g}}=400 \mathrm{GeV}$ up to $22 \%$ for $m_{\tilde{g}}=1000 \mathrm{GeV}$ for light squarks. For heavy squarks, the EW contributions are only at the percent level due to the interplay of positive EW corrections in the LL and RR case and negative EW corrections in the LR case, suppressing the EW contributions by one order in magnitude.

We can understand the smallness of the EW contribution for high squark and low gluino masses by having a closer look at the interplay of the tree-level EW and NLO EW contributions. The ratio $\Delta \sigma^{\mathrm{NLO}} \mathrm{EW} / \Delta \sigma^{\text {tree } \mathrm{EW}}$ for inclusive squark-squark production is shown in figure 7 (a). As one can see, the NLO EW corrections become more important for larger ratios $m_{\tilde{q}} / m_{\tilde{g}}$ and reach the same size as the tree-level EW contributions for about $m_{\tilde{q}} / m_{\tilde{g}} \gtrsim 1.5$, depending on the precise value of the gluino mass. This is due to the fact that the LR contribution becomes more relevant for increasing $m_{\tilde{q}} / m_{\tilde{g}}$, see figure 7 (b). Owing to the suppressed tree-level contributions, the EW contributions to LR production are negative and partially compensate the positive yield from LL and RR production. 


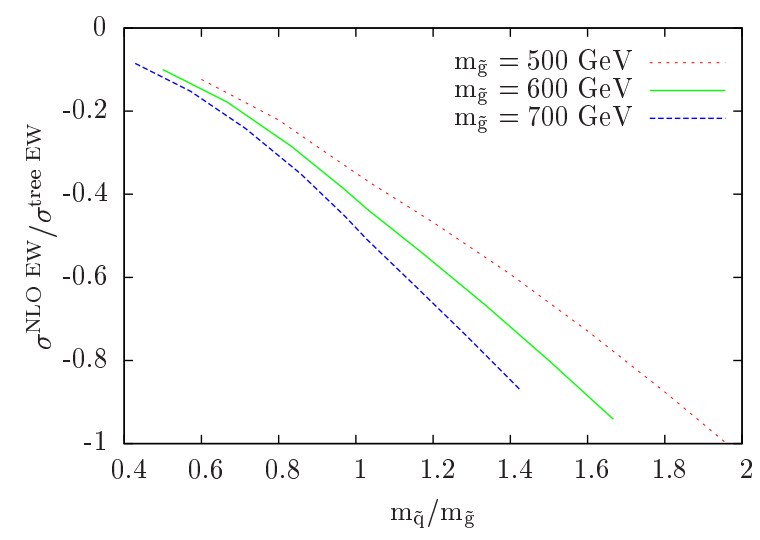

(a)

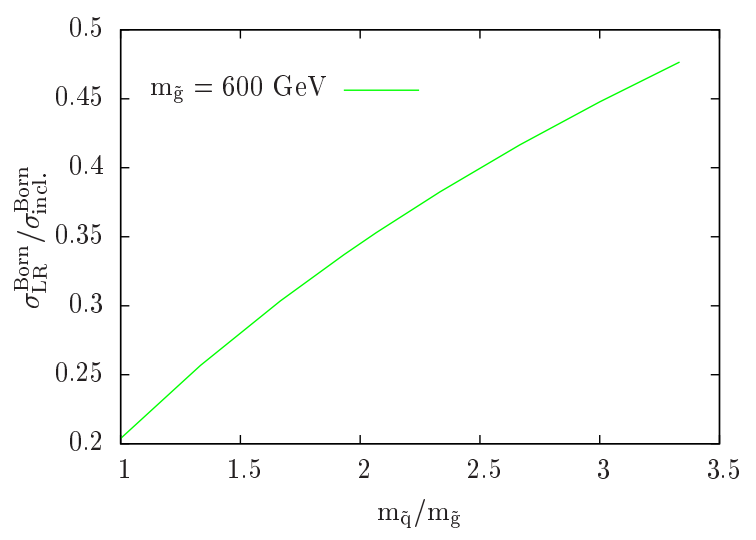

(b)

Figure 7. (a) Ratio of NLO EW to tree-level EW contributions for inclusive squark-squark production. (b) Relative contribution of LR final states to the inclusive Born cross section for a fixed gluino mass $m_{\tilde{g}}=600 \mathrm{GeV}$.

Figure 7 (a) also confirms our observation from the SPS2 scenario that the NLO EW corrections compensate the tree-level EW contributions in the inclusive cross section, cf. table 4, which seems to be a generic feature in scenarios with the squark heavier than the gluino.

\subsection{Differential distributions}

Here, we illustrate the results for the SPS1a' scenario. In figures 8, 9, and 10 we consider the differential distributions of the EW contributions with respect to various kinematical variables. In the left panels, the tree-level EW contributions and the three gauge-invariant subsets of NLO EW contributions (EW-type corrections, QCD-type corrections, real quark radiation), as well as the summed EW contributions are shown. In the right panels, the impact of the EW contributions relative to the Born cross section, $\delta$, is given.

Figure 8 refers to the distribution with respect to the transverse momentum $\mathrm{p}_{\mathrm{T}}$ of the squark with highest $\mathrm{p}_{\mathrm{T}}$. The tree-level EW contributions are always positive with a maximum at about $250 \mathrm{GeV}$ and dominate the sum over a wide range of the phase-space for LL, RR, and inclusive squark-squark production. Again, they are suppressed for LR production. The interplay of the NLO EW contributions is more complicated. For all processes, the real quark radiation is small and mostly negative. For LL production, large cancellations among the EW- and QCD-type corrections occur. As a result, the relative yield is dominated from the tree-level contributions in the small- $\mathrm{p}_{\mathrm{T}}$ region where it is large and positive (up to $25 \%$ ). For higher values of $\mathrm{p}_{\mathrm{T}}$, the relative corrections turn negative and grow up to $-10 \%$. In case of RR production the EW-type corrections are suppressed from the chirality and the QCD-type corrections are more important. However the relative EW contributions in total do not exceed a few percent. Finally in the LR case, the QCD-type corrections are negligible since they are related to QCD-EW interferences. The dominant contribution arises here from the EW-type corrections. The relative contributions are always negative, between $-2 \%$ for small values of $\mathrm{p}_{\mathrm{T}}$ and up to $-10 \%$ in the high- $\mathrm{p}_{\mathrm{T}}$ region. It is important to note that even though the relative NLO EW contributions to 

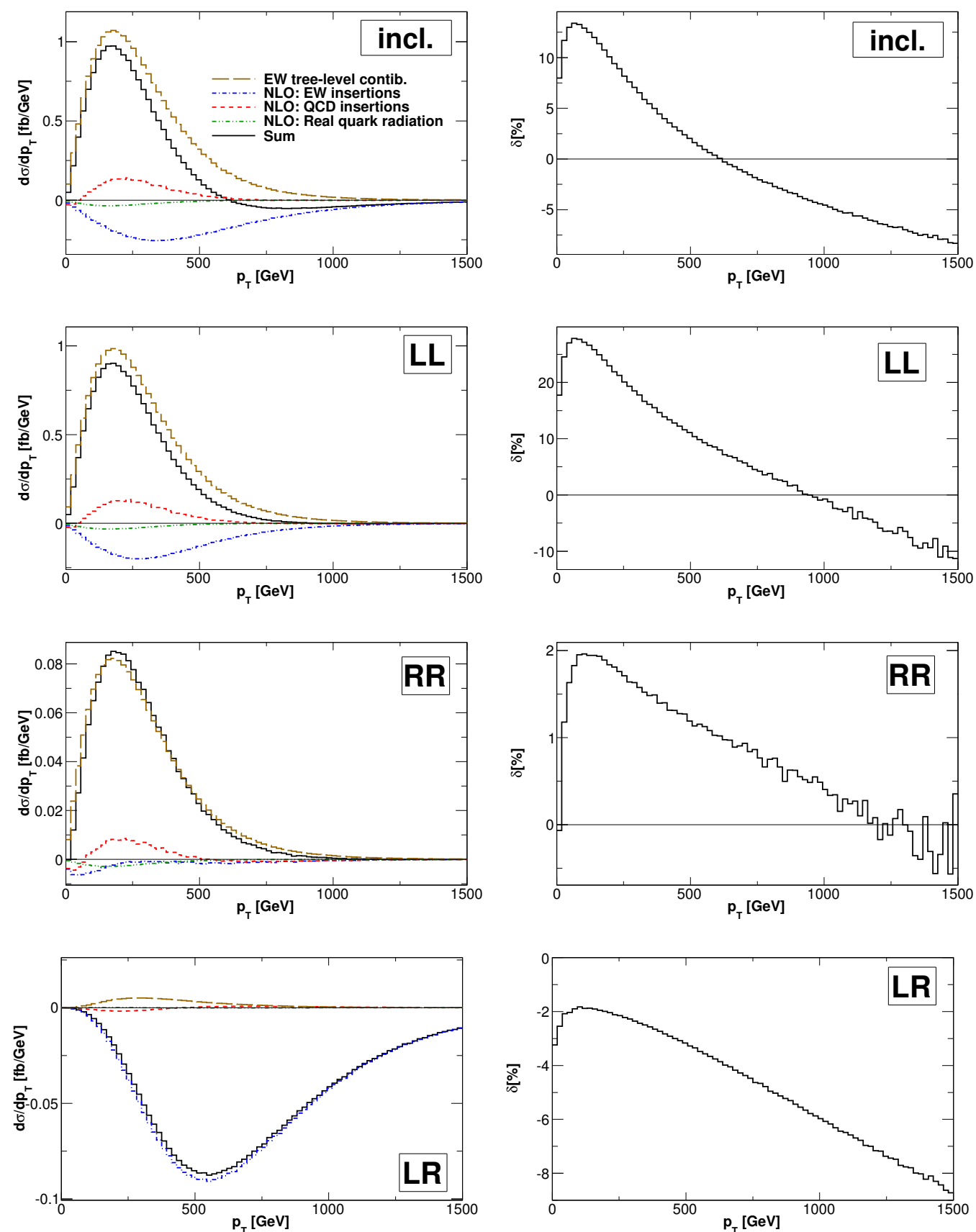

Figure 8. Transverse momentum distributions for squark-squark production at the LHC within the SPS1a' scenario. Shown are the tree-level and NLO EW cross section contributions (left) and the impact of EW contributions relative to the QCD Born cross section (right) for inclusive $\tilde{q} \tilde{q}^{\prime}$ production (top), production of two left-handed squarks $\tilde{q}_{L} \tilde{q}_{L}^{\prime}$ (second), production of two righthanded squarks $\tilde{q}_{R} \tilde{q}_{R}^{\prime}$ (third), and non-diagonal $\tilde{q}_{L} \tilde{q}_{R}^{\prime}$ production (bottom). Charge conjugated processes are included.

the integrated cross section are comparable for LL and LR production, cf. table 3, they originate from distinct sources and the differential distributions differ strongly. 

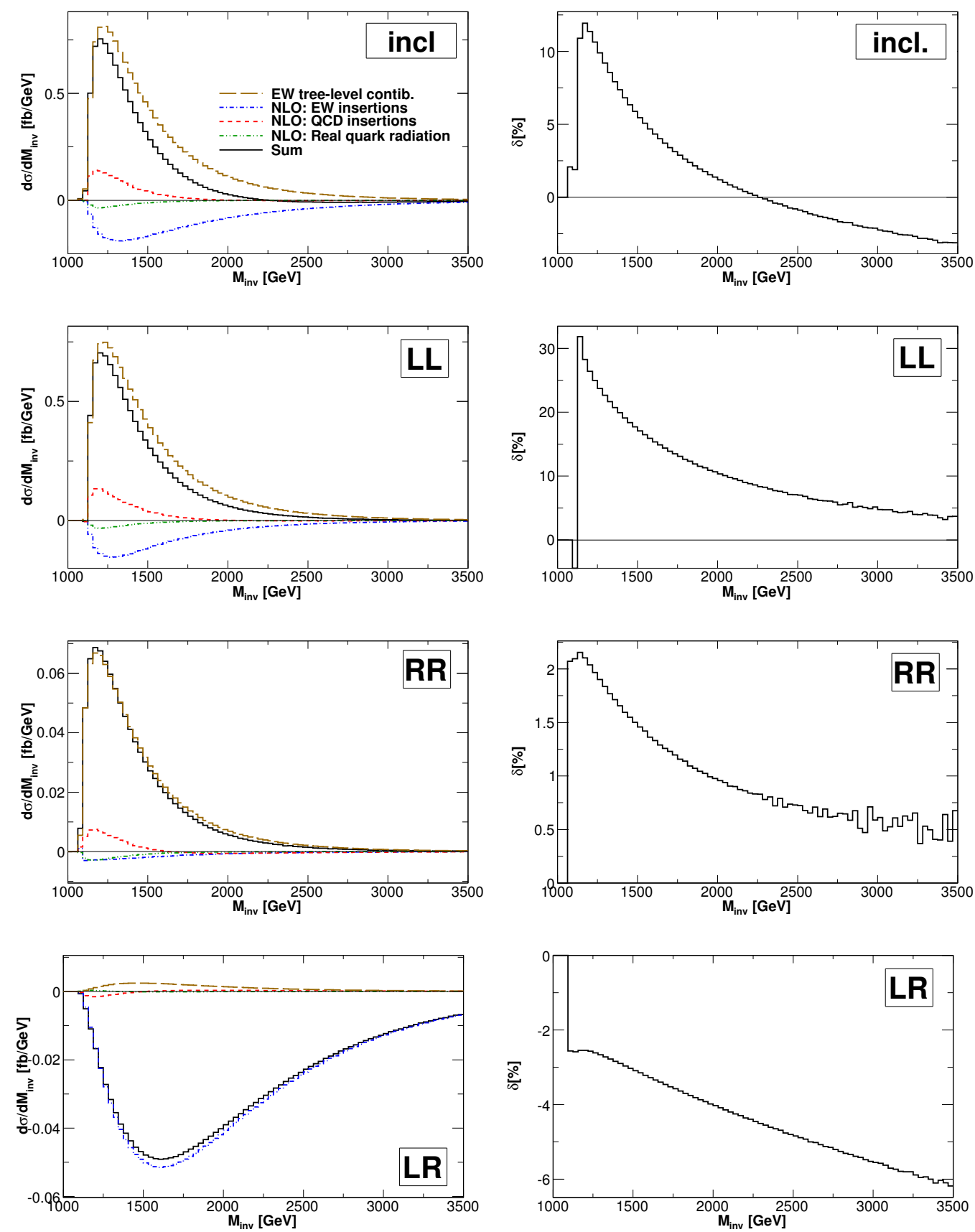

Figure 9. Invariant mass distributions for squark-squark production at the LHC within the SPS1a' scenario. Shown are the tree-level and NLO EW cross section contributions (left) and the impact of EW contributions relative to the QCD Born cross section (right) for inclusive $\tilde{q} \tilde{q}^{\prime}$ production (top), production of two left-handed squarks $\tilde{q}_{L} \tilde{q}_{L}^{\prime}$ (second), production of two right-handed squarks $\tilde{q}_{R} \tilde{q}_{R}^{\prime}$ (third), and non-diagonal $\tilde{q}_{L} \tilde{q}_{R}^{\prime}$ production (bottom). Charge conjugated processes are included.

In figure 9 the distributions with respect to the invariant mass of the squark pair, $M_{\mathrm{inv}}=\left(p_{3}+p_{4}\right)^{2}$, are shown. The interplay of the various subsets of EW contributions is similar as for the $\mathrm{p}_{\mathrm{T}}$ distributions. For LL and RR production, the EW tree-level contri- 

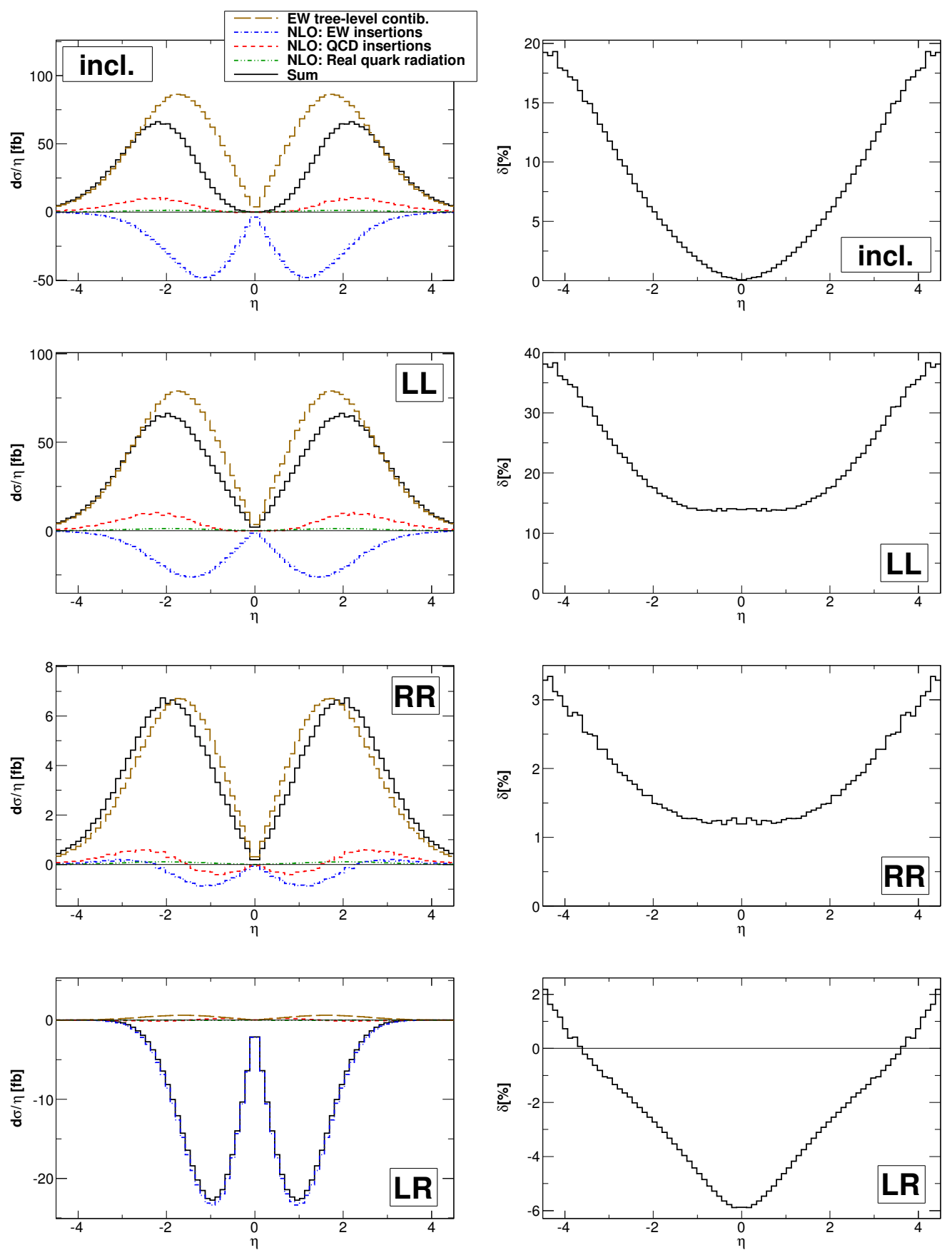

Figure 10. Pseudo-rapidity distributions for squark-squark production at the LHC within the SPS1a' scenario. Shown are the tree-level and NLO EW cross section contributions (left) and the impact of EW contributions relative to the QCD Born cross section (right) for inclusive $\tilde{q} \tilde{q}^{\prime}$ production (top), production of two left-handed squarks $\tilde{q}_{L} \tilde{q}_{L}^{\prime}$ (second), production of two righthanded squarks $\tilde{q}_{R} \tilde{q}_{R}^{\prime}$ (third), and non-diagonal $\tilde{q}_{L} \tilde{q}_{R}^{\prime}$ production (bottom). Charge conjugated processes are included. 
butions are dominant and peak at around $M_{\mathrm{inv}} \approx 1200 \mathrm{GeV}$. They shift the relative EW corrections to positive values, up to $30 \%$ in the low- $M_{\text {inv }}$ region for LL production. In case of non-diagonal LR production, where the EW tree-level contributions are suppressed, the relative corrections are negative and grow up to $-5 \%$ for the intermediate and high-energy region. Finally, in the inclusive case, we find a strong energy dependence of the relative EW corrections, ranging from $+10 \%$ for $M_{\mathrm{inv}} \approx 1200 \mathrm{GeV}$ to $-5 \%$ for $M_{\mathrm{inv}}>3500 \mathrm{GeV}$.

In figure 10 we present the pseudo-rapidity distributions, where always the squark with the higher absolute value of the pseudo-rapidity $\eta$ (in the laboratory frame) is considered. All EW contributions are vanishing in the central $\eta \approx 0$ region. The characteristics of the rapidity gap in the distributions depend on the precise quantity considered and is enhanced by our choice of referring to the larger $\eta$. The tree-level contributions peak at around $|\eta|=2$ and dominate the total result, if present. The distributions for EW-type and QCD-type NLO corrections differ in sign and shape from each other, leading to large cancellations over a wide phase-space range. In total, the EW contributions alter the LO distributions by up to $20-40 \%$ for LL and up to $10-20 \%$ for inclusive squark-squark production in the strong forward region for $|\eta|>2$.

Up to now, our discussion has only been for inclusive combinations of final-state squarks for given chiralities. To get further insight on the cross section, we show in table 6 the cross section divided up into the various subprocesses for squark-squark production within the SPS1a' scenario. Again, anti-particles are included. Owing to the degenerate masses of first- and second-generation squarks, we do not distinguish between final states that result from exchanging both squarks with their first or second generation counterpart, i.e. $\tilde{u}_{L} \tilde{u}_{L}$ production also includes $\tilde{c}_{L} \tilde{c}_{L}$ production, etc.. This reduces the number of distinct subprocesses from 36 down to 22 . The contributions to $\Delta \sigma^{\text {tree } \mathrm{EW}}$ are always positive and are largest for $\tilde{u}_{L} \tilde{d}_{L}$ production due to the interference of gluino and chargino exchange diagrams and constitute $57 \%$ of the inclusive tree-level EW contribution, see also table 3. One even finds that the inclusive tree-level EW contribution is given to $98 \%$ by only five processes, namely $\tilde{u}_{L} \tilde{u}_{L}, \tilde{u}_{R} \tilde{u}_{R}, \tilde{d}_{L} \tilde{d}_{L}, \tilde{u}_{L} \tilde{d}_{L}$ and $\tilde{u}_{L} \tilde{s}_{L}$. The contributions to $\Delta \sigma^{\mathrm{NLO}} \mathrm{EW}$ are mostly negative, reducing the importance of EW contributions. In contrast to the tree-level EW case, many processes contribute with a significant amount to the inclusive NLO EW contribution of the cross section. Especially for processes with squarks of different generations, $\Delta \sigma^{\mathrm{EW}}$ is mostly dominated by NLO EW contributions. The size of the NLO EW contributions is often reduced due to the interplay of QCD-type and EWtype corrections as shown in figure 11 in the case of $\tilde{u}_{L} \tilde{d}_{L}$ production. The different types of NLO EW corrections partially cancel. Furthermore, the sum contains corrections of positive and negative sign, leading to an integrated result $\Delta \sigma^{\mathrm{NLO}}$ EW that is considerably smaller than the corrections affecting the LO result in various phase-space regions.

\section{Conclusions}

We have studied the hadronic production of two squarks or two anti-squarks within the MSSM, including tree-level EW and NLO EW cross section contributions of $\mathcal{O}\left(\alpha_{s} \alpha+\alpha^{2}\right)$ and $\mathcal{O}\left(\alpha_{s}^{2} \alpha\right)$, respectively. In contrast to pure QCD computations, one has to treat pro- 


\begin{tabular}{|c|c|c|c|c|c|c|}
\hline SPS1a' & $\begin{array}{l}\sigma^{\text {Born }} \\
\mathcal{O}\left(\alpha_{s}^{2}\right)\end{array}$ & $\begin{array}{c}\Delta \boldsymbol{\sigma}^{\text {tree }} \mathbf{E W} \\
\mathcal{O}\left(\alpha_{s} \alpha+\alpha^{2}\right)\end{array}$ & $\begin{array}{c}\Delta \boldsymbol{\sigma}^{\text {NLO EW }} \\
\mathcal{O}_{\left(\alpha_{s}^{2} \alpha\right)}\end{array}$ & $\delta^{\text {tree } \mathrm{EW}}$ & $\delta^{\mathrm{NLO}} \mathrm{EW}$ & $\delta^{\mathrm{EW}}$ \\
\hline$\tilde{\boldsymbol{u}}_{L} \tilde{\boldsymbol{u}}_{L}$ & $486.8(3)$ & $93.78(5)$ & $-30.5(2)$ & $19.27 \%$ & $-6.26 \%$ & $13.00 \%$ \\
\hline$\tilde{d}_{L} \tilde{d}_{L}$ & $143.83(8)$ & $29.18(2)$ & $-9.85(6)$ & $20.29 \%$ & $-6.85 \%$ & $13.44 \%$ \\
\hline$\tilde{u}_{L} \tilde{d}_{L}$ & $692.6(7)$ & $234.8(2)$ & $-9.5(6)$ & $33.90 \%$ & $-1.38 \%$ & $32.52 \%$ \\
\hline$\tilde{\boldsymbol{u}}_{L} \tilde{\boldsymbol{s}}_{L}$ & $211.3(2)$ & $17.95(3)$ & $-8.53(1)$ & $8.50 \%$ & $-4.04 \%$ & $4.46 \%$ \\
\hline$\tilde{u}_{L} \tilde{c}_{L}$ & $102.96(8)$ & $1.864(2)$ & $-8.885(7)$ & $1.81 \%$ & $-8.63 \%$ & $-6.82 \%$ \\
\hline$\tilde{d}_{L} \tilde{s}_{L}$ & $80.19(6)$ & $1.390(2)$ & $-7.526(4)$ & $1.73 \%$ & $-9.39 \%$ & $-7.65 \%$ \\
\hline$\tilde{\boldsymbol{u}}_{\boldsymbol{R}} \tilde{\boldsymbol{u}}_{\boldsymbol{R}}$ & $537.1(4)$ & $28.58(2)$ & $-4.44(8)$ & $5.32 \%$ & $-0.83 \%$ & $4.49 \%$ \\
\hline$\tilde{d}_{R} \tilde{d}_{R}$ & $173.1(1)$ & $2.414(2)$ & $-0.318(7)$ & $1.39 \%$ & $-0.18 \%$ & $1.21 \%$ \\
\hline$\tilde{u}_{R} \tilde{d}_{R}$ & $799.1(6)$ & $0.4458(8)$ & $3.41(3)$ & $0.06 \%$ & $0.43 \%$ & $0.48 \%$ \\
\hline$\tilde{u}_{R} \tilde{s}_{R}$ & $253.0(2)$ & $0.1276(2)$ & $1.36(1)$ & $0.05 \%$ & $0.54 \%$ & $0.59 \%$ \\
\hline$\tilde{\boldsymbol{u}}_{\boldsymbol{R}} \tilde{\boldsymbol{c}}_{\boldsymbol{R}}$ & $118.95(9)$ & $0.2365(4)$ & $-1.337(8)$ & $0.20 \%$ & $-1.12 \%$ & $-0.93 \%$ \\
\hline$\tilde{d}_{R} \tilde{s}_{R}$ & $100.65(8)$ & $0.0126(1)$ & $-0.281(2)$ & $0.01 \%$ & $-0.28 \%$ & $-0.27 \%$ \\
\hline$\tilde{\boldsymbol{u}}_{\boldsymbol{L}} \tilde{\boldsymbol{u}}_{\boldsymbol{R}}$ & $629.7(4)$ & $1.288(1)$ & $-26.41(4)$ & $0.20 \%$ & $-4.19 \%$ & $-3.99 \%$ \\
\hline$\tilde{d}_{L} \tilde{d}_{R}$ & $165.49(9)$ & $0.0792(1)$ & $-7.027(4)$ & $0.05 \%$ & $-4.25 \%$ & $-4.20 \%$ \\
\hline$\tilde{\boldsymbol{u}}_{L} \tilde{\boldsymbol{d}}_{\boldsymbol{R}}$ & $328.5(2)$ & $0.1720(1)$ & $-12.30(1)$ & $0.05 \%$ & $-3.75 \%$ & $-3.69 \%$ \\
\hline$\tilde{u}_{R} \tilde{d}_{L}$ & $321.4(2)$ & $0.6026(6)$ & $-13.81(2)$ & $0.19 \%$ & $-4.30 \%$ & $-4.11 \%$ \\
\hline$\tilde{\boldsymbol{u}}_{L} \tilde{\boldsymbol{s}}_{R}$ & $82.26(4)$ & $0.0450(1)$ & $-2.809(3)$ & $0.05 \%$ & $-3.42 \%$ & $-3.36 \%$ \\
\hline$\tilde{\boldsymbol{u}}_{\boldsymbol{R}} \tilde{\boldsymbol{s}}_{\boldsymbol{L}}$ & $79.90(4)$ & $0.1556(1)$ & $-3.167(4)$ & $0.19 \%$ & $-3.96 \%$ & $-3.77 \%$ \\
\hline$\tilde{\boldsymbol{u}}_{L} \tilde{\boldsymbol{c}}_{\boldsymbol{R}}$ & $38.08(2)$ & $0.0832(1)$ & $-1.388(2)$ & $0.22 \%$ & $-3.65 \%$ & $-3.43 \%$ \\
\hline$\tilde{\boldsymbol{u}}_{\boldsymbol{R}} \tilde{\boldsymbol{c}}_{\boldsymbol{L}}$ & $38.08(2)$ & $0.0832(1)$ & $-1.388(2)$ & $0.22 \%$ & $-3.65 \%$ & $-3.44 \%$ \\
\hline$\tilde{d}_{L} \tilde{s}_{R}$ & $30.24(2)$ & $0.0149(1)$ & $-1.2015(9)$ & $0.05 \%$ & $-3.97 \%$ & $-3.92 \%$ \\
\hline$\tilde{d}_{R} \tilde{s}_{L}$ & $30.24(2)$ & $0.0149(1)$ & $-1.2015(9)$ & $0.05 \%$ & $-3.97 \%$ & $-3.92 \%$ \\
\hline
\end{tabular}

Table 6. Hadronic cross section for squark-squark production at the LHC within the SPS1a scenario for $\sqrt{S}=14 \mathrm{TeV}$. Charge conjugated processes are included. $\tilde{u} \tilde{u}$ final states include $\tilde{c} \tilde{c}, \tilde{d} \tilde{d}$ include $\tilde{s} \tilde{s}$, and $\tilde{u} \tilde{s}$ includes $\tilde{c} \tilde{d}$. All cross sections are given in femtobarn (fb).

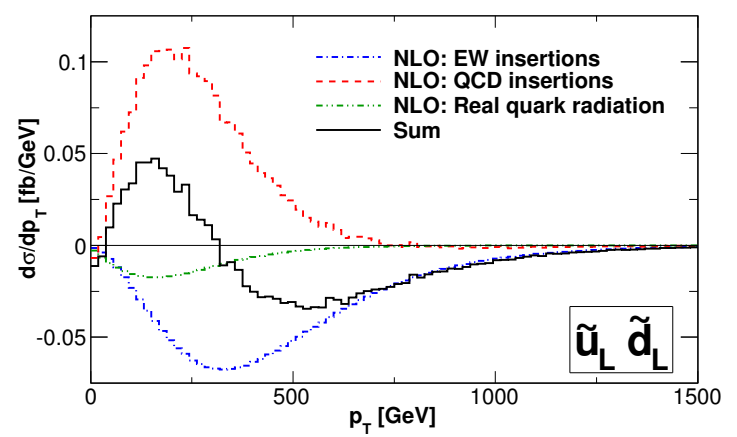

(a)

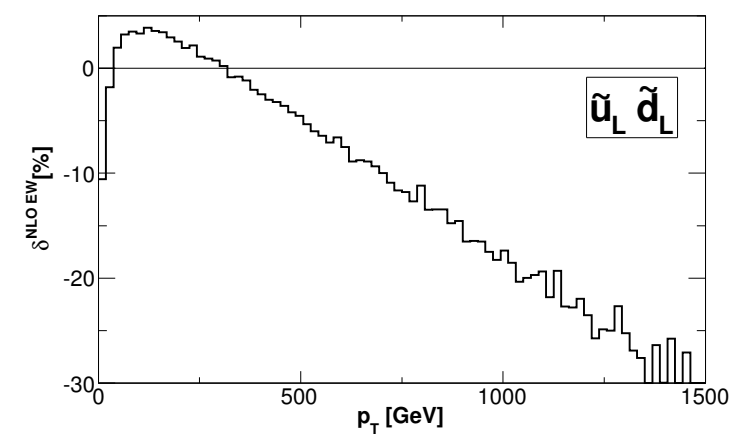

(b)

Figure 11. (a) Transverse momentum distribution of the hardest squark for $\tilde{u}_{L} \tilde{d}_{L}$ production within the SPS1a' scenario. Strong cancellations occur between the different contributions at NLO EW. (b) Relative NLO EWcontribution, defined as the ratio of $d \sigma^{\mathrm{NLO}} \mathrm{EW} / d p_{T}$ and $d \sigma^{\mathrm{Born}} / d p_{T}$. 
cesses with squarks of different flavor or chirality separately. At $\mathcal{O}\left(\alpha_{s}^{2} \alpha\right)$, numerous QCDEW interference terms occur. Virtual corrections arise from the interference contributions of tree-level QCD amplitudes and mixed EW-QCD one-loop diagrams, as well as from the interference of tree-level EW and pure-QCD one-loop amplitudes. Bremsstrahlung corrections comprise real photon, real gluon, and real quark radiation processes.

We have performed a detailed numerical analysis for two left-handed squarks $\left(\tilde{q}_{L} \tilde{q}_{L}\right)$, two right-handed squarks $\left(\tilde{q}_{R} \tilde{q}_{R}\right)$, and one left-handed and one right-handed squark $\left(\tilde{q}_{L} \tilde{q}_{R}\right)$, as well as for inclusive squark-squark $(\tilde{q} \tilde{q})$ production at the LHC. The tree-level EW contributions are largest for $\tilde{q}_{L} \tilde{q}_{L}$ production, where they are enhanced by $\mathcal{O}\left(\alpha_{s} \alpha\right)$ interference terms and can easily reach the $20 \%$ level. The interference contributions are suppressed for $\tilde{q}_{R} \tilde{q}_{R}$ production from the chiral couplings and vanish for $\tilde{q}_{L} \tilde{q}_{R}$ production in the limit of no L-R mixing. At NLO, partial cancellations occur among the various EW contributions. As a result, the integrated cross section is reduced by the NLO EW contributions by a few percent for most subprocesses. The interplay of tree-level and NLO EW contributions is not universal and depends sensitively on the ratio of squark and gluino masses as well as on their absolute values. The full EW contributions affect the integrated cross section for inclusive squark-squark production at the percent level (about 5\% in SPS1a ${ }^{\prime}$ and SPS5, $-1 \%$ in SPS2). In the distributions, however, the EW contributions range from $-10 \%$ to $20 \%$ and even larger values for exclusive $\tilde{q}_{L} \tilde{q}_{L}$ production.

\section{Acknowledgments}

E. M. and M. T. thank their former home institution MPI for Physics for hospitality during the final stages of the work. M. T. is supported by the DOE grant DE-FG02-95ER40896. E.M.'s research is supported by the European Research Council under Advanced Investigator Grant ERC-AdG-228301. This work was supported in part by the European Community's Marie-Curie Research Training Network under contract MRTN-CT-2006-035505 'Tools and Precision Calculations for Physics Discoveries at Colliders' (HEPTOOLS).

\section{A Tree-level cross sections}

In this appendix, we give explicit expressions for the tree-level differential cross sections eq. (2.5)-eq. (2.6) for all squark-squark production subprocesses. We closely follow [24] and express the color- and spin-averaged squared $t$ - and $u$-channel matrix elements and their interference in terms of the following functions,

$$
\begin{aligned}
\Phi\left(\tilde{\xi}_{1}, \tilde{\xi}_{2}, \tilde{q}_{\alpha}, \tilde{q}_{\beta}^{\prime}\right)= & \frac{1}{4} c_{\Phi}\left(\tilde{\xi}_{1}, \tilde{\xi}_{2}\right) \frac{1}{\hat{t}-m_{\tilde{\xi}_{1}}^{2}} \frac{1}{\hat{t}-m_{\tilde{\xi}_{2}}^{2}}\left[A\left(\tilde{\xi}_{1}, \tilde{\xi}_{2}, \tilde{q}_{\alpha}, \tilde{q}_{\beta}^{\prime}\right)\right. \\
& \left.\times\left(\hat{t} \hat{u}-m_{\tilde{q}_{\alpha}}^{2} m_{\tilde{q}_{\beta}^{\prime}}^{2}\right)+B\left(\tilde{\xi}_{1}, \tilde{\xi}_{2}, \tilde{q}_{\alpha}, \tilde{q}_{\beta}^{\prime}\right) m_{\tilde{\xi}_{1}} m_{\tilde{\xi}_{2}} \hat{s}\right], \\
\Theta\left(\tilde{\xi}_{1}, \tilde{\xi}_{2}, \tilde{q}_{\alpha}, \tilde{q}_{\beta}^{\prime}\right)= & \frac{1}{4} c_{\Theta}\left(\tilde{\xi}_{1}, \tilde{\xi}_{2}\right) \frac{1}{\hat{u}-m_{\tilde{\xi}_{1}}^{2}} \frac{1}{\hat{u}-m_{\tilde{\xi}_{2}}^{2}}\left[C\left(\tilde{\xi}_{1}, \tilde{\xi}_{2}, \tilde{q}_{\alpha}, \tilde{q}_{\beta}^{\prime}\right)\right. \\
& \left.\times\left(\hat{t} \hat{u}-m_{\tilde{q}_{\alpha}}^{2} m_{\tilde{q}_{\beta}^{\prime}}^{2}\right)+D\left(\tilde{\xi}_{1}, \tilde{\xi}_{2}, \tilde{q}_{\alpha}, \tilde{q}_{\beta}^{\prime}\right) m_{\tilde{\xi}_{1}} m_{\tilde{\xi}_{2}} \hat{s}\right], \\
\Psi\left(\tilde{\xi}_{1}, \tilde{\xi}_{2}, \tilde{q}_{\alpha}, \tilde{q}_{\beta}^{\prime}\right)= & \frac{1}{4} c_{\Psi}\left(\tilde{\xi}_{1}, \tilde{\xi}_{2}\right) \frac{1}{\hat{t}-m_{\tilde{\xi}_{1}}^{2}} \frac{1}{\hat{u}-m_{\tilde{\xi}_{2}}^{2}} F\left(\tilde{\xi}_{1}, \tilde{\xi}_{2}, \tilde{q}_{\alpha}, \tilde{q}_{\beta}^{\prime}\right) m_{\tilde{\xi}_{1}} m_{\tilde{\xi}_{2}} \hat{s}
\end{aligned}
$$




\begin{tabular}{|c|c|c|c|}
\hline$\tilde{\boldsymbol{\xi}}_{\mathbf{1}}, \tilde{\boldsymbol{\xi}}_{\mathbf{2}}$ & $\boldsymbol{c}_{\boldsymbol{\Phi}}\left(\tilde{\boldsymbol{\xi}}_{\mathbf{1}}, \tilde{\boldsymbol{\xi}}_{\mathbf{2}}\right)$ & $\boldsymbol{c}_{\boldsymbol{\Theta}}\left(\tilde{\boldsymbol{\xi}}_{\mathbf{1}}, \tilde{\boldsymbol{\xi}}_{\mathbf{2}}\right)$ & $\boldsymbol{c}_{\boldsymbol{\Psi}}\left(\tilde{\boldsymbol{\xi}}_{\mathbf{1}}, \tilde{\boldsymbol{\xi}}_{\mathbf{2}}\right)$ \\
\hline$\tilde{g}, \tilde{g}$ & $2 / 9$ & $2 / 9$ & $-2 / 27$ \\
\hline$\tilde{\chi}, \tilde{\chi}$ & 1 & 1 & $1 / 3$ \\
\hline$\tilde{g}, \tilde{\chi}$ & 0 & 0 & $4 / 9$ \\
\hline
\end{tabular}

Table 7. Color factors in eqs. (A.1)-(A.3), with $\tilde{\chi}$ denoting any neutralino $\tilde{\chi}_{k}^{0}$ or chargino $\tilde{\chi}_{k}^{ \pm}$.
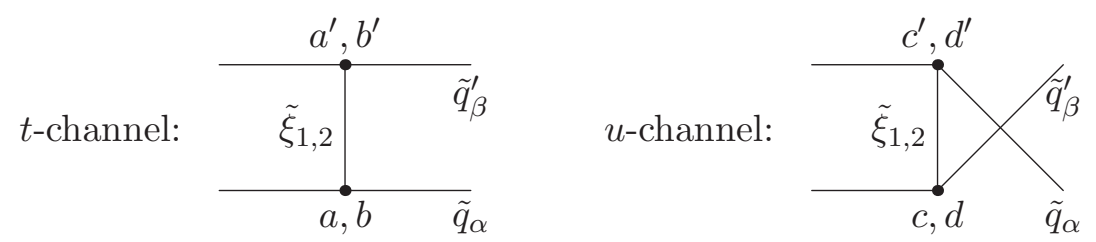

Figure 12. Notations for the couplings in the tree-level formulas in appendix A. Labels $a, c$ refer to the couplings to left-handed squarks, $b$ and $d$ to that of right-handed squarks. Couplings at the upper and lower vertex, respectively, are denoted by distinct labels for convenience.

Here, $\tilde{\xi}_{1,2} \in\left\{\tilde{g}, \tilde{\chi}^{0}, \tilde{\chi}^{ \pm}\right\}$denote the exchanged particle in the $t$ - or $u$-channel, respectively, and $c_{\{\Phi, \Theta, \Psi\}}$ are color matrices which are summarized in table 7 . Note that the color factors $c_{\Psi}(\tilde{\chi}, \tilde{\chi})$ differ from [24].

The coupling constants are collected in the abbreviations $A, B, C, D, F$, as given by

$$
\begin{aligned}
& A\left(\tilde{\xi}_{1}, \tilde{\xi}_{2}, \tilde{q}_{\alpha}, \tilde{q}_{\beta}^{\prime}\right)=a_{\tilde{\xi}_{1}, \tilde{q}_{\alpha}} a_{\tilde{\xi}_{2}, \tilde{q}_{\alpha}}^{*} b_{\tilde{\xi}_{1}, \tilde{q}_{\beta}^{\prime}}^{\prime} b_{\tilde{\xi}_{2}, \tilde{q}_{\beta}^{\prime}}^{\prime *}+a_{\tilde{\xi}_{1}, \tilde{q}_{\beta}^{\prime}}^{\prime} a_{\tilde{\xi}_{2}, \tilde{q}_{\beta}^{\prime}}^{\prime *} b_{\tilde{\xi}_{1}, \tilde{q}_{\alpha}} b_{\tilde{\xi}_{2}, \tilde{q}_{\alpha}}^{*}, \\
& B\left(\tilde{\xi}_{1}, \tilde{\xi}_{2}, \tilde{q}_{\alpha}, \tilde{q}_{\beta}^{\prime}\right)=a_{\tilde{\xi}_{1}, \tilde{q}_{\alpha}} a_{\tilde{\xi}_{2}, \tilde{q}_{\alpha}}^{*} a_{\tilde{\xi}_{1}, \tilde{q}_{\beta}^{\prime}}^{\prime} a_{\tilde{\xi}_{2}, \tilde{q}_{\beta}^{\prime}}^{\prime *}+b_{\tilde{\xi}_{1}, \tilde{q}_{\alpha}} b_{\tilde{\xi}_{2}, \tilde{q}_{\alpha}}^{*} b_{\tilde{\xi}_{1}, \tilde{q}_{\beta}^{\prime}}^{\prime} b_{\tilde{\xi}_{2}, \tilde{q}_{\beta}^{\prime}}^{\prime *}, \\
& C\left(\tilde{\xi}_{1}, \tilde{\xi}_{2}, \tilde{q}_{\alpha}, \tilde{q}_{\beta}^{\prime}\right)=c_{\tilde{\xi}_{1}, \tilde{q}_{\beta}^{\prime}} c_{\tilde{\xi}_{2}, \tilde{q}_{\beta}^{\prime}}^{*} d_{\tilde{\xi}_{1}, \tilde{q}_{\alpha}}^{\prime} d_{\tilde{\xi}_{2}, \tilde{q}_{\alpha}}^{\prime *}+c_{\tilde{\xi}_{1}, \tilde{q}_{\alpha}}^{\prime} c_{\tilde{\xi}_{2}, \tilde{q}_{\alpha}}^{\prime *} d_{\tilde{\xi}_{1}, \tilde{q}_{\beta}^{\prime}} d_{\tilde{\xi}_{2}, \tilde{q}_{\beta}^{\prime}}^{*}, \\
& D\left(\tilde{\xi}_{1}, \tilde{\xi}_{2}, \tilde{q}_{\alpha}, \tilde{q}_{\beta}^{\prime}\right)=c_{\tilde{\xi}_{1}, \tilde{q}_{\beta}} c_{\tilde{\xi}_{2}, \tilde{q}_{\beta}^{\prime}}^{*} c_{\tilde{\xi}_{1}, \tilde{q}_{\alpha}}^{\prime} c_{\tilde{\xi}_{2}, \tilde{q}_{\alpha}}^{\prime *}+d_{\tilde{\xi}_{1}, \tilde{q}_{\beta}^{\prime}} d_{\tilde{\xi}_{2}, \tilde{q}_{\beta}^{\prime}}^{*} d_{\tilde{\xi}_{1}, \tilde{q}_{\alpha}}^{\prime} d_{\tilde{\xi}_{2}, \tilde{q}_{\alpha}}^{\prime *}, \\
& F\left(\tilde{\xi}_{1}, \tilde{\xi}_{2}, \tilde{q}_{\alpha}, \tilde{q}_{\beta}^{\prime}\right)=a_{\tilde{\xi}_{1}, \tilde{q}_{\alpha}} c_{\tilde{\xi}_{2}, \tilde{q}_{\beta}^{\prime}}^{*} a_{\tilde{\xi}_{1}, \tilde{q}_{\beta}^{\prime}}^{\prime} c_{\tilde{\xi}_{2}, \tilde{q}_{\alpha}}^{\prime *}+b_{\tilde{\xi}_{1}, \tilde{q}_{\alpha}} d_{\tilde{\xi}_{2}, \tilde{q}_{\beta}^{\prime}}^{*} b_{\tilde{\xi}_{1}, \tilde{q}_{\beta}^{\prime}}^{\prime} d_{\tilde{\xi}_{2}, \tilde{q}_{\alpha}}^{\prime *},
\end{aligned}
$$

where the notation refers to the labels as listed in figure 12. Finally the explicit coupling constants $a_{\tilde{\xi}_{i}, \tilde{q}_{\alpha}}, b_{\tilde{\xi}_{i}, \tilde{q}_{\alpha}}, \ldots$ are given in table 8 .

For the differential cross sections, we refer to the three classes of subprocesses introduced in eq. (2.3). $\alpha, \beta=\{\mathrm{L}, \mathrm{R}\}$ label the chirality of the squarks, $k, l$ label the four (two) mass eigenstates of neutralinos (charginos).

- $P P \rightarrow \tilde{q}_{\alpha} \tilde{q}_{\beta}$ (two squarks of the same flavor)

The partonic process for this class of processes is $q q \rightarrow \tilde{q}_{\alpha} \tilde{q}_{\beta}$, i.e. all quarks and squarks are of the same flavor. The differential cross sections at 


\begin{tabular}{|c|c|c|}
\hline$\tilde{\boldsymbol{\xi}}_{\boldsymbol{i}}, \tilde{\boldsymbol{q}}_{\boldsymbol{\alpha}}$ & $\boldsymbol{a}, \boldsymbol{a}^{\prime}, \boldsymbol{c}, \boldsymbol{c}^{\prime}$ & $\boldsymbol{b}, \boldsymbol{b}^{\prime}, \boldsymbol{d}, \boldsymbol{d}^{\prime}$ \\
\hline$\tilde{\chi}_{k}^{0}, \tilde{u}_{\alpha}$ & $-\frac{i e}{\sqrt{2} s_{w}}\left(\frac{1}{3} \frac{s_{w}}{c_{w}} N_{k 1}^{*}+N_{k 2}^{*}\right) \delta_{L \alpha}$ & $\frac{4 i e}{3 \sqrt{2} c_{w}} N_{k 1} \delta_{R \alpha}$ \\
\hline$\tilde{\chi}_{k}^{0}, \tilde{d}_{\alpha}$ & $-\frac{i e}{\sqrt{2} s_{w}}\left(\frac{1}{3} \frac{s_{w}}{c_{w}} N_{k 1}^{*}-N_{k 2}^{*}\right) \delta_{L \alpha}$ & $-\frac{2 i e}{3 \sqrt{2} c_{w}} N_{k 1} \delta_{R \alpha}$ \\
\hline$\tilde{\chi}_{k}^{ \pm}, \tilde{u}_{\alpha}$ & $-\frac{i e}{s_{w}} V_{k 1}^{*} \delta_{L \alpha}$ & 0 \\
\hline$\tilde{\chi}_{k}^{ \pm}, \tilde{d}_{\alpha}$ & $-\frac{i e}{s_{w}} U_{k 1}^{*} \delta_{L \alpha}$ & 0 \\
\hline$\tilde{g}, \tilde{u}_{\alpha}$ & $-\sqrt{2} i \hat{g}_{s} \delta_{L \alpha}$ & $\sqrt{2} i \hat{g}_{s} \delta_{R \alpha}$ \\
\hline$\tilde{g}, \tilde{d}_{\alpha}$ & $-\sqrt{2} i \hat{g}_{s} \delta_{L \alpha}$ & $\sqrt{2} i \hat{g}_{s} \delta_{R \alpha}$ \\
\hline
\end{tabular}

Table 8. Coupling constants $a_{\tilde{\xi}_{i}, \tilde{q}_{\alpha}}, b_{\tilde{\xi}_{i}, \tilde{q}_{\alpha}}, \ldots$ for exchange particle $\tilde{\xi}_{i}$ and produced light-flavor squark $\tilde{q}_{\alpha}$, following the conventions of [35]. L-R mixing of the squark mass eigenstates is neglected. $N, U, V$ are the unitary matrices diagonalizing the neutralino and chargino mass matrix, respectively and $s_{w}=\sin \theta_{w}$ and $c_{w}=\cos \theta_{w}$.

$$
\begin{gathered}
\mathcal{O}\left(\alpha_{s}^{2}\right), \mathcal{O}\left(\alpha^{2}\right), \mathcal{O}\left(\alpha_{s} \alpha\right) \text { read, according to the notation introduced in section } 2, \\
d \hat{\sigma}^{2,0}=\left\{\Phi\left(\tilde{g}, \tilde{g}, \tilde{q}_{\alpha}, \tilde{q}_{\beta}\right)+\Theta\left(\tilde{g}, \tilde{g}, \tilde{q}_{\alpha}, \tilde{q}_{\beta}\right)+2 \operatorname{Re}\left\{\Psi\left(\tilde{g}, \tilde{g}, \tilde{q}_{\alpha}, \tilde{q}_{\beta}\right)\right\}\right\} \frac{d \hat{t}}{16 \pi \hat{s}^{2}}, \\
d \hat{\sigma}^{0,2}=\sum_{k, l=1}^{4}\left\{\Phi\left(\tilde{\chi}_{k}^{0}, \tilde{\chi}_{l}^{0}, \tilde{q}_{\alpha}, \tilde{q}_{\beta}\right)+\Theta\left(\tilde{\chi}_{k}^{0}, \tilde{\chi}_{l}^{0}, \tilde{q}_{\alpha}, \tilde{q}_{\beta}\right)\right. \\
\left.\quad+2 \operatorname{Re}\left\{\Psi\left(\tilde{\chi}_{k}^{0}, \tilde{\chi}_{l}^{0}, \tilde{q}_{\alpha}, \tilde{q}_{\beta}\right)\right\}\right\} \frac{d \hat{t}}{16 \pi \hat{s}^{2}}, \\
d \hat{\sigma}^{1,1}=\sum_{k=1}^{4} 2 \operatorname{Re}\left\{\Psi\left(\tilde{g}, \tilde{\chi}_{k}^{0}, \tilde{q}_{\alpha}, \tilde{q}_{\beta}\right)+\Psi\left(\tilde{\chi}_{k}^{0}, \tilde{g}, \tilde{q}_{\alpha}, \tilde{q}_{\beta}\right)\right\} \frac{d \hat{t}}{16 \pi \hat{s}^{2}} .
\end{gathered}
$$

As can be seen from the couplings in table 8, the interference terms $\Psi$ and thus in particular the interference contribution $d \hat{\sigma}^{1,1}$ are only present for diagonal squarksquark production (i.e. $\alpha=\beta$ ). This is a result from the absence of L-R mixing for the light-flavor squarks.

- $P P \rightarrow \tilde{q}_{\alpha} \tilde{q}_{\beta}^{\prime}$ (two squarks of different flavor in the same $S U$ (2) doublet)

The only contributing partonic process is $q q^{\prime} \rightarrow \tilde{q}_{\alpha} \tilde{q}_{\beta}^{\prime}$, with $q^{\prime}$ being the $\mathrm{SU}(2)$ partner of $q$. The tree-level contributions to the cross section read:

$$
\begin{aligned}
d \hat{\sigma}^{2,0}= & \Phi\left(\tilde{g}, \tilde{g}, \tilde{q}_{\alpha}, \tilde{q}_{\beta}^{\prime}\right) \frac{d \hat{t}}{16 \pi \hat{s}^{2}}, \\
d \hat{\sigma}^{0,2}= & \left\{\sum_{k, l=1}^{4} \Phi\left(\tilde{\chi}_{k}^{0}, \tilde{\chi}_{l}^{0}, \tilde{q}_{\alpha}, \tilde{q}_{\beta}^{\prime}\right)+\sum_{k, l=1}^{2} \Theta\left(\tilde{\chi}_{k}^{ \pm}, \tilde{\chi}_{l}^{ \pm}, \tilde{q}_{\alpha}, \tilde{q}_{\beta}^{\prime}\right)\right. \\
& \left.+\sum_{k=1}^{4} \sum_{l=1}^{2} 2 \operatorname{Re}\left\{\Psi\left(\tilde{\chi}_{l}^{0}, \tilde{\chi}_{k}^{ \pm}, \tilde{q}_{\alpha}, \tilde{q}_{\beta}^{\prime}\right)\right\}\right\} \frac{d \hat{t}}{16 \pi \hat{s}^{2}}, \\
d \hat{\sigma}^{1,1}= & \sum_{k=1}^{2} 2 \operatorname{Re}\left\{\Psi\left(\tilde{g}, \tilde{\chi}_{k}^{ \pm}, \tilde{q}_{\alpha}, \tilde{q}_{\beta}^{\prime}\right)\right\} \frac{d \hat{t}}{16 \pi \hat{s}^{2}} .
\end{aligned}
$$


In this case, the interference terms are related to chargino-mediated diagrams and thus the interference contribution $d \hat{\sigma}^{1,1}$ is only non-zero for the production of two left-handed squarks (i.e. $\alpha=\beta=L$ ).

- $P P \rightarrow \tilde{q}_{\alpha} \tilde{q}_{\beta}^{\prime}$ (two squarks in different doublets)

This class describes the production of two squarks of different flavor and of different generation, arising from the partonic process $q q^{\prime} \rightarrow \tilde{q}_{\alpha} \tilde{q}_{\beta}^{\prime}$, with $q \neq q^{\prime}$. The tree-level cross sections read as follows,

$$
\begin{aligned}
d \hat{\sigma}^{2,0}= & \Phi\left(\tilde{g}, \tilde{g}, \tilde{q}_{\alpha}, \tilde{q}_{\beta}^{\prime}\right) \frac{d \hat{t}}{16 \pi \hat{s}^{2}}, \\
d \hat{\sigma}^{0,2}= & \left\{\sum_{k, l=1}^{4} \Phi\left(\tilde{\chi}_{k}^{0}, \tilde{\chi}_{l}^{0}, \tilde{q}_{\alpha}, \tilde{q}_{\beta}^{\prime}\right)+\delta_{q u} \delta_{q^{\prime} s} \delta_{\tilde{q} \tilde{d}} \delta_{\tilde{q}^{\prime} \tilde{c}} \sum_{k, l=1}^{2} \Phi\left(\tilde{\chi}_{k}^{ \pm}, \tilde{\chi}_{l}^{ \pm}, \tilde{q}_{\alpha}, \tilde{q}_{\beta}^{\prime}\right)\right. \\
& \left.+\delta_{q c} \delta_{q^{\prime} d} \delta_{\tilde{q} \tilde{u}} \delta_{\tilde{q}^{\prime} \tilde{s}} \sum_{k, l=1}^{2} \Phi\left(\tilde{\chi}_{k}^{ \pm}, \tilde{\chi}_{l}^{ \pm}, \tilde{q}_{\alpha}, \tilde{q}_{\beta}^{\prime}\right)\right\} \frac{d \hat{t}}{16 \pi \hat{s}^{2}}, \\
d \hat{\sigma}^{1,1}= & 0 .
\end{aligned}
$$

Here, two additional chargino-mediated partonic processes $\left(u s \rightarrow \tilde{d}_{L} \tilde{c}_{L}\right.$ and $c d \rightarrow$ $\left.\tilde{u}_{L} \tilde{s}_{L}\right)$ can give an $\mathcal{O}\left(\alpha^{2}\right)$ contribution. The $\mathcal{O}\left(\alpha_{s} \alpha\right)$ interference contribution vanishes for this class of processes.

\section{B Bremsstrahlung integrals}

Here we give the soft and collinear singular integrals appearing in the phase space integration of the real radiation processes, with either a photon, gluon or (anti-)quark radiated.

\section{B.1 Soft and collinear photon bremsstrahlung}

The cross section for real photon emission factorizes in the soft limit from the original cross section without photon emission,

$$
\left.d \hat{\sigma}_{12 \rightarrow 34 \gamma}^{2,1}\right|_{\text {soft }}=-\frac{\alpha}{2 \pi}\left\{\sum_{i, j=1 ; i<j}^{4} e_{i} e_{j} \sigma_{i} \sigma_{j} \mathcal{I}_{i j}\right\} d \hat{\sigma}_{12 \rightarrow 34}^{2,0},
$$

where now the considered process is given as a subscript for clarification and particles are labeled by $i=1 \ldots 4$ according to the definition of momenta $p_{i}$ in eqs. (2.2) and (3.2). $e_{i}$ is the charge of the $i$ th particle and $\sigma_{i}= \pm 1$ depending on whether the particle is incoming or outgoing, respectively. The phase-space integrals $\mathcal{I}_{i j}=\mathcal{I}_{j i}$ are universal and well-known [48]. In the limit of vanishing initial-state masses (and two massive final-state 
particles), they are given by

$$
\begin{aligned}
\mathcal{I}_{i i}=\ln \left(\frac{4(\Delta E)^{2}}{\lambda^{2}}\right)+\ln \left(\frac{m_{i}^{2}}{\hat{s}_{12}}\right) & \text { for } i=\{1,2\}, \\
\mathcal{I}_{i i}=\ln \left(\frac{4(\Delta E)^{2}}{\lambda^{2}}\right)+\frac{1}{\beta_{i}} \ln \left(\frac{1-\beta_{i}}{1+\beta_{i}}\right) & \text { for } i=\{3,4\}, \\
\mathcal{I}_{12}=\sum_{i=1,2}\left[\ln \left(\frac{\hat{s}_{12}}{m_{i}^{2}}\right) \ln \left(\frac{4(\Delta E)^{2}}{\lambda^{2}}\right)-\frac{1}{2} \ln ^{2}\left(\frac{\hat{s}_{12}}{m_{i}^{2}}\right)-\frac{\pi^{2}}{3}\right], & \\
\mathcal{I}_{34}= & \frac{1}{v_{34}} \sum_{i=3,4}\left[\ln \left(\frac{1+\beta_{i}}{1-\beta_{i}}\right) \ln \left(\frac{4(\Delta E)^{2}}{\lambda^{2}}\right)-2 \operatorname{Li}_{2}\left(\frac{2 \beta_{i}}{1+\beta_{i}}\right)-\frac{1}{2} \ln ^{2}\left(\frac{1-\beta_{i}}{1+\beta_{i}}\right)\right], \\
\mathcal{I}_{i j}= & \ln \left(\frac{\hat{s}_{i j}^{2}}{m_{i}^{2} m_{j}^{2}}\right) \ln \left(\frac{4(\Delta E)^{2}}{\lambda^{2}}\right)-\frac{1}{2} \ln ^{2}\left(\frac{\hat{s}_{12}}{m_{i}^{2}}\right)-\frac{1}{2} \ln ^{2}\left(\frac{1-\beta_{i}}{1+\beta_{i}}\right)-\frac{\pi^{2}}{3} \\
& -2 \operatorname{Li}_{2}\left(1-\frac{2 p_{i}^{0} p_{j}^{0}}{\hat{s}_{i j}}\left(1+\beta_{j}\right)\right)-2 \operatorname{Li}_{2}\left(1-\frac{2 p_{i}^{0} p_{j}^{0}}{\hat{s}_{i j}}\left(1-\beta_{j}\right)\right) \quad \text { for } \begin{array}{l}
i=\{1,2\}, \\
j=\{3,4\},
\end{array}
\end{aligned}
$$

with $\hat{s}_{i j}=2 p_{i} \cdot p_{j}, \beta_{i}=\left|p_{i}\right| / p_{i}^{0}, v_{i j}=\sqrt{1-4 m_{i} m_{j} / \hat{s}_{i j}^{2}}, \Delta E=\sqrt{\hat{s}_{12}} \delta_{s} / 2 . \lambda$ is the fictitious photon mass.

The partonic cross section in the collinear region can be written in terms of a convolution integral

$$
\left.d \hat{\sigma}_{12 \rightarrow 34 \gamma}^{2,1}(\hat{s})\right|_{\text {coll }}=\frac{\alpha\left(e_{q}^{2}+e_{q^{\prime}}^{2}\right)}{2 \pi} \int_{z_{0}}^{1-\delta_{s}} \mathrm{~d} z \kappa_{q q}(z, \hat{s}) d \hat{\sigma}_{12 \rightarrow 34}^{2,0}(z \hat{s})
$$

where $z_{0}$ and $\kappa_{q q}$ are given by

$$
z_{0}=\left(m_{\tilde{q}}^{2}+m_{\tilde{q}^{\prime}}^{2}\right) / \hat{s}, \quad \kappa_{q q}(z, \hat{s})=\frac{1+z^{2}}{1-z} \ln \left(\frac{\hat{s} \delta_{\theta}}{2 m_{q}}\right)-\frac{2 z}{1-z} .
$$

The upper integration bound is lowered by $\delta_{s}$ to avoid double counting of the soft regime.

\section{B.2 Soft and collinear gluon bremsstrahlung}

The bremsstrahlung integrals for soft gluon emission are the same as those for the photonic case, but in addition one has to take the color correlation of the amplitudes into account. We use the following notation to keep track of the color factors.

Let $\left|c_{1}, \ldots, c_{m}\right\rangle$ denote a complete color basis. The colored matrix element with $m$ external particles $\mathrm{P}_{i}$ carrying momentum $p_{i}$ and color $c_{i}$ is then given by

$$
\mathcal{M}_{12 \rightarrow 3 \ldots m}^{i, j c_{1} \ldots c_{m}}=\left\langle c_{1}, \ldots, c_{m} \mid \mathcal{M}_{12 \rightarrow 3 \ldots m}^{i, j}\right\rangle .
$$

For the color structure in the case of real gluon emission it is convenient to associate a color charge $\mathbf{T}_{i}$ with the emission of a gluon of color $a$ from parton $i$. The action of this color charge onto the color space is given by

$$
\left\langle c_{1} \ldots c_{i} \ldots c_{m}\left|\mathbf{T}_{i}\right| b_{1} \ldots b_{i} \ldots b_{m}\right\rangle=\delta_{c_{1} b_{1}} \ldots T_{c_{i} b_{i}}^{a} \ldots \delta_{c_{m} b_{m}} .
$$


Explicit expressions for the $T_{k l}^{a}$ and the color charge algebra are given in [49]. The real gluon emission in the soft limit is then given by:

$$
\left.d \hat{\sigma}_{12 \rightarrow 34 g}\right|_{\text {soft }}=-\frac{\alpha_{s}}{2 \pi}\left\{\sum_{i, j=1 ; i<j}^{4} \mathcal{I}_{i j} \mathcal{F}_{i j}\right\} \frac{d t}{16 \pi \hat{s}^{2}},
$$

where the phase space integrals $\mathcal{I}_{i j}$ are given in eq. (B.3) ${ }^{3}$ and $\mathcal{F}_{i j}$ denote color correlated amplitudes. At $\mathcal{O}\left(\alpha_{s}^{2} \alpha\right), \mathcal{F}_{i j}$ are given by

$$
\begin{aligned}
\mathcal{F}_{i j} & =2 \operatorname{Re}\left\{\left\langle\mathcal{M}_{12 \rightarrow 34}^{0,1}\left|\mathbf{T}_{i} \mathbf{T}_{j}\right| \mathcal{M}_{12 \rightarrow 34}^{1,0}\right\rangle\right\} \\
& =2 \operatorname{Re}\left\{\left[\mathcal{M}_{12 \rightarrow 34}^{0,1 c_{1} \ldots b_{i} \ldots b_{j} \ldots c_{4}}\right]^{*} T_{b_{i} c_{i}}^{a} T_{b_{j} c_{j}}^{a} \mathcal{M}_{12 \rightarrow 34}^{1,0 c_{1} \ldots c_{i} \ldots c_{j} \ldots c_{4}}\right\} .
\end{aligned}
$$

In the case of squark-squark production the tree-level amplitude can be decomposed according to their color structure as

$$
\begin{array}{rlrl}
\mathcal{M}_{12 \rightarrow 34}^{i, j c_{1} c_{2} c_{3} c_{4}} & =\delta_{c_{1} c_{3}} \delta_{c_{2} c_{4}} \mathcal{M}_{1}^{i, j}+\delta_{c_{1} c_{4}} \delta_{c_{2} c_{2}} \mathcal{M}_{2}^{i, j}, & (i, j)=(1,0),(0,1), \\
\mathcal{M}_{1}^{0,1} & =\mathcal{M}_{T}^{0,1}, & \mathcal{M}_{1}^{1,0} & =\frac{1}{2}\left(\mathcal{M}_{U}^{1,0}-\frac{1}{3} \mathcal{M}_{T}^{1,0}\right), \\
\mathcal{M}_{2}^{0,1} & =\mathcal{M}_{U}^{0,1}, & \mathcal{M}_{2}^{1,0} & =\frac{1}{2}\left(\mathcal{M}_{T}^{1,0}-\frac{1}{3} \mathcal{M}_{U}^{1,0}\right),
\end{array}
$$

where $\mathcal{M}_{U, T}^{i, j}$ are the amplitudes corresponding to the $u$-channel and $t$-channel diagrams, respectively. In this case the color correlated amplitudes $\mathcal{F}_{i j}$ are given by

$$
\begin{aligned}
& \mathcal{F}_{12}=\mathcal{F}_{34}=4\left[\left(\mathcal{M}_{1}^{0,1}\right)^{*} \mathcal{M}_{2}^{1,0}+\mathcal{M}_{2}^{0,1 *} \mathcal{M}_{1}^{1,0}\right] \\
& \mathcal{F}_{13}=\mathcal{F}_{24}=-12\left(\mathcal{M}_{1}^{0,1}\right)^{*} \mathcal{M}_{1}^{1,0}-4\left[\left(\mathcal{M}_{1}^{0,1}\right)^{*} \mathcal{M}_{2}^{1,0}+\left(\mathcal{M}_{2}^{0,1}\right)^{*} \mathcal{M}_{1}^{1,0}\right], \\
& \mathcal{F}_{14}=\mathcal{F}_{23}=-4\left[\left(\mathcal{M}_{1}^{0,1}\right)^{*} \mathcal{M}_{2}^{1,0}+\left(\mathcal{M}_{2}^{0,1}\right)^{*} \mathcal{M}_{1}^{1,0}\right]-12\left(\mathcal{M}_{2}^{0,1}\right)^{*} \mathcal{M}_{2}^{1,0} \\
& \mathcal{F}_{i i}=12\left[\left(\mathcal{M}_{1}^{0,1}\right)^{*} \mathcal{M}_{1}^{1,0}+\left(\mathcal{M}_{2}^{0,1}\right)^{*} \mathcal{M}_{2}^{1,0}\right]+4\left[\left(\mathcal{M}_{1}^{0,1}\right)^{*} \mathcal{M}_{2}^{1,0}+\left(\mathcal{M}_{2}^{0,1}\right)^{*} \mathcal{M}_{1}^{1,0}\right],
\end{aligned}
$$

where in the last case $i=1, \ldots, 4$.

The partonic cross section in the collinear region is again given by a convolution integral, similar to eq. (B.4),

$$
\left.d \hat{\sigma}_{12 \rightarrow 34 g}^{2,1}(\hat{s})\right|_{\text {coll }}=\frac{\alpha_{s} C_{F}}{\pi} \int_{z_{0}}^{1-\delta_{s}} d z \kappa_{q q}(z, \hat{s}) d \hat{\sigma}_{12 \rightarrow 34}^{1,1}(z \hat{s})
$$

with $C_{F}=4 / 3 . z_{0}$ and $\kappa_{q q}$ are defined in eq. (B.4).

\section{B.3 Collinear quark bremsstrahlung}

For real quark emission, only initial-state collinear singularities arise. The partonic cross section in the collinear region is given by a convolution integral,

$$
\left.d \hat{\sigma}_{12 \rightarrow 34 \bar{q}}^{2,1}(\hat{s})\right|_{\text {coll }}=\frac{\alpha_{s} T_{F}}{2 \pi} \int_{z_{0}}^{1} \mathrm{~d} z \kappa_{q g}(z, \hat{s}) d \hat{\sigma}_{12 \rightarrow 34}^{1,1}(z \hat{s}),
$$

\footnotetext{
${ }^{3}$ In this case $\lambda$ is the fictitious gluon mass.
} 


\begin{tabular}{|c|c|c|c||c|c|c|}
\hline SPS1a $^{\prime}$ & $\begin{array}{c}\boldsymbol{\sigma}^{\text {Born }} \\
\mathcal{O}\left(\alpha_{s}^{2}\right)\end{array}$ & $\begin{array}{c}\boldsymbol{\Delta} \boldsymbol{\sigma}^{\text {tree EW }} \\
\mathcal{O}\left(\alpha_{s} \alpha+\alpha^{2}\right)\end{array}$ & $\begin{array}{c}\boldsymbol{\Delta} \boldsymbol{\sigma}^{\text {NLO EW }} \\
\mathcal{O}\left(\alpha_{s}^{2} \alpha\right)\end{array}$ & $\boldsymbol{\delta}^{\text {tree EW }}$ & $\boldsymbol{\delta}^{\text {NLO EW }}$ & $\boldsymbol{\delta}^{\text {EW }}$ \\
\hline$\tilde{\boldsymbol{q}}_{\boldsymbol{L}} \tilde{\boldsymbol{q}}_{\boldsymbol{L}}^{\prime}$ & $342.0(1)$ & $89.81(3)$ & $-6.1(1)$ & $26.3 \%$ & $-1.8 \%$ & $24.5 \%$ \\
\hline$\tilde{\boldsymbol{q}}_{\boldsymbol{R}} \tilde{\boldsymbol{q}}_{\boldsymbol{R}}^{\prime}$ & $408.7(1)$ & $9.78(5)$ & $0.40(2)$ & $2.4 \%$ & $0.1 \%$ & $2.5 \%$ \\
\hline$\tilde{\boldsymbol{q}}_{\boldsymbol{L}} \tilde{\boldsymbol{q}}_{\boldsymbol{R}}^{\prime}$ & $274.79(7)$ & $0.4571(2)$ & $-9.661(7)$ & $0.2 \%$ & $-3.5 \%$ & $-3.3 \%$ \\
\hline \hline$\tilde{\boldsymbol{q}} \tilde{\boldsymbol{q}}^{\prime}$ & $1025.5(2)$ & $100.05(3)$ & $-15.3(1)$ & $9.8 \%$ & $-1.5 \%$ & $8.3 \%$ \\
\hline
\end{tabular}

Table 9. Hadronic cross sections in femtobarn (fb) for squark-squark production at the LHC within the SPS1a' scenario for $\sqrt{S}=7 \mathrm{TeV}$. Notation as in table 3 .

with $T_{F}=1 / 2 . \kappa_{q g}$ is given by

$$
\kappa_{q g}=\left(z^{2}+(1-z)^{2}\right) \ln \left(\frac{\hat{s} \delta_{\theta}(1-z)^{2}}{2 m_{q}^{2}}\right)+2 z(1-z)
$$

while $z_{0}$ is defined according to Eq (B.4).

\section{Hadronic cross sections for $\sqrt{S}=7 \mathrm{TeV}$}

In table 9 we give the results for the hadronic cross sections for inclusive squark-squark production at the LHC for $\sqrt{S}=7 \mathrm{TeV}$ within the SPS1a' scenario. All other inputs are chosen as in table 3 and section 4 .

The integrated cross sections amount about $15 \%-25 \%$ of their values at $\sqrt{S}=14 \mathrm{TeV}$. The relative importance of tree-level EW contributions increases by a few percent points while the relative NLO EW corrections are less important. All subprocesses are quarkquark initiated and the corresponding luminosities are reduced to a similar extent. However the relative importance of each of the 36 subprocesses changes a little. At Born level the contribution of down-quark induced processes to the inclusive Born cross section is smaller than at $14 \mathrm{TeV}$, whereas their impact at NLO is increased. In particular the $\tilde{u}_{\alpha} \tilde{d}_{\alpha}$ channels give large and positive contributions at NLO EW. As a consequence, the NLO EW corrections for inclusive RR production are now positive and those for LL production are reduced to $-2 \%$. In summary, the full EW contributions alter the LO cross section for inclusive squark-squark production at the LHC for $\sqrt{S}=7 \mathrm{TeV}$ by $8 \%$ within the SPS1a' scenario.

\section{Feynman diagrams}

In this appendix, we list all parton-level Feynman diagrams for the EW contributions to the generic process $q q^{\prime} \rightarrow \tilde{q}_{\alpha} \tilde{q}_{\beta}^{\prime}$ with $q, q^{\prime}=\{u, d, c, s\}$. The complete list of LO Feynman diagrams is given in figure 1 . 


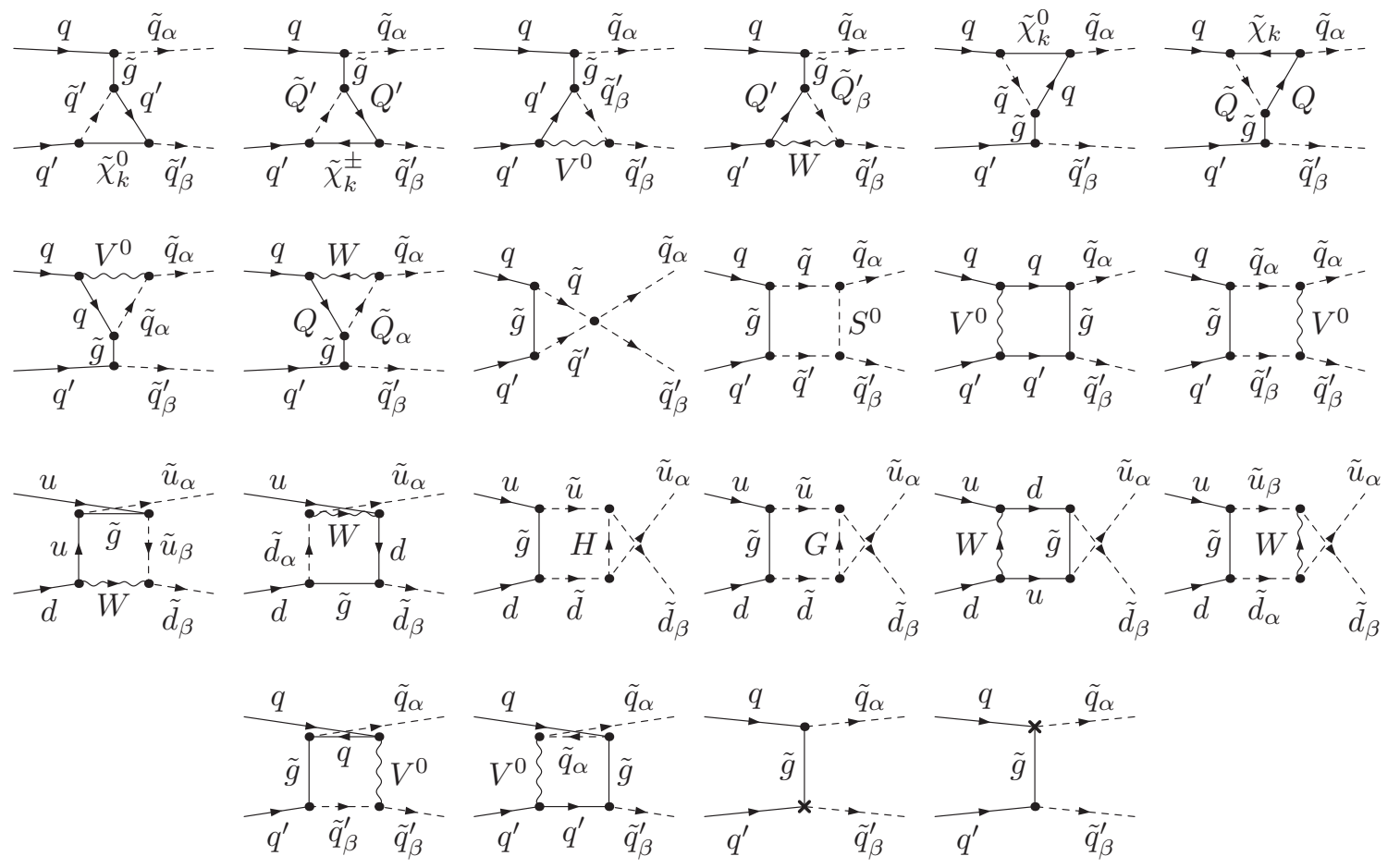

Figure 13. Virtual corrections (I): EW one-loop insertions into QCD Born diagrams. $Q$ and $Q^{\prime}$ denote the $\mathrm{SU}(2)$ partner of quark $q$ and $q^{\prime}$, respectively. We use generic labels $V^{0}=\gamma, Z$ and $S^{0}=h^{0}, H^{0}, G^{0}, A^{0}$. If the chirality of an internal squark is not specified, it can be any. The diagram containing the four-squark vertex has to be taken at $\mathcal{O}\left(\alpha_{s} \alpha\right)$. The diagrams in the third line contribute only for $u=\{u, c\}, d=\{d, s\}$. The last two diagrams contain the counter terms, whose renormalization constants have to be evaluated at $\mathcal{O}(\alpha)$. For $q=q^{\prime}$ crossed diagrams have to be taken into account. 

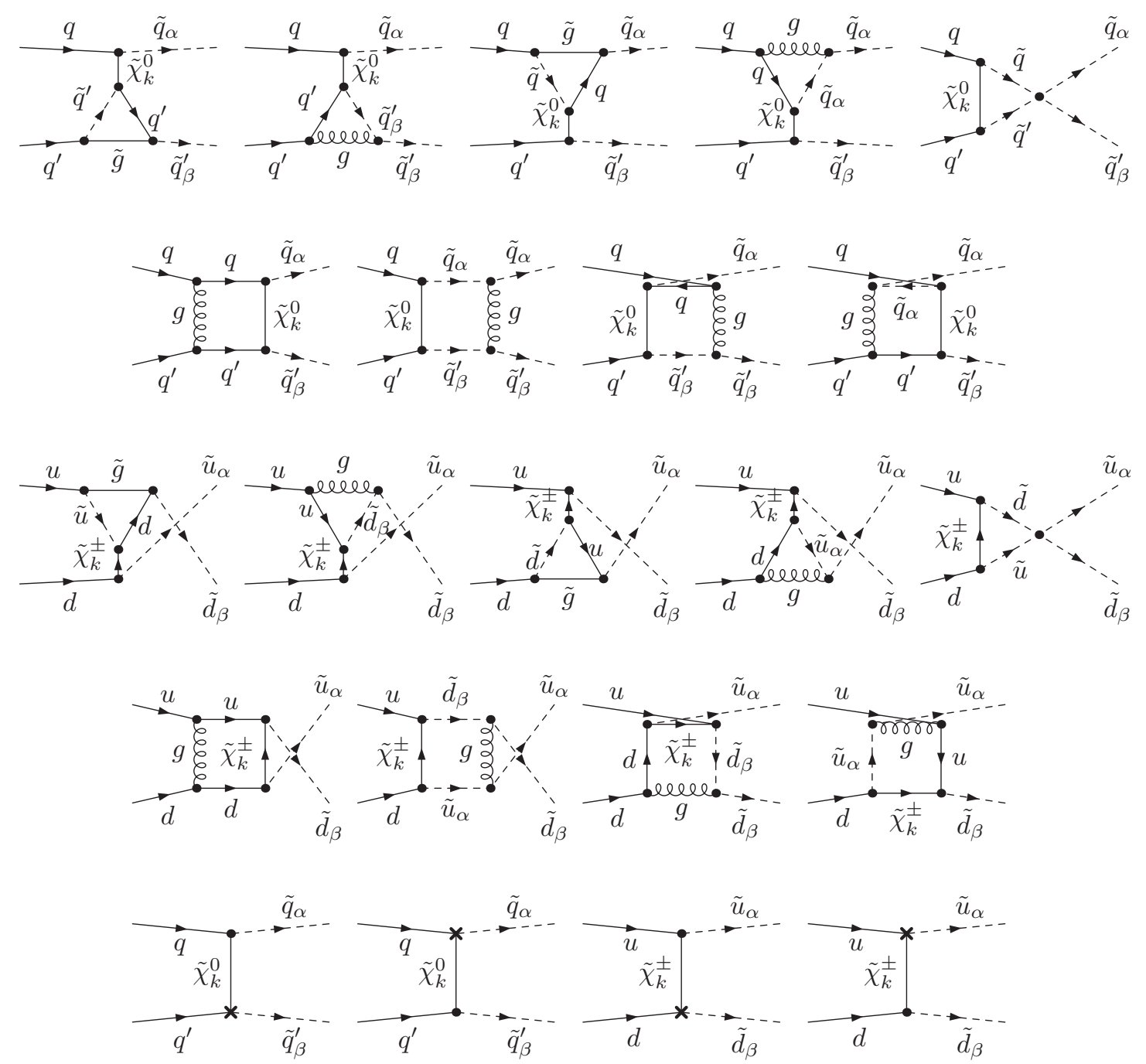

Figure 14. Virtual corrections (II): QCD one-loop insertions into EW Born diagrams. For $q=q^{\prime}$ crossed diagrams have to be taken into account. The diagrams containing the four squark interaction vertex have to be evaluated at $\mathcal{O}\left(\alpha_{s} \alpha\right)$. The chargino-mediated diagrams only contribute for $u=$ $\{u, c\}, d=\{d, s\}$. The renormalization constants appearing in the counterterm diagrams (last line) have to be evaluated at $\mathcal{O}\left(\alpha_{s}\right)$. 

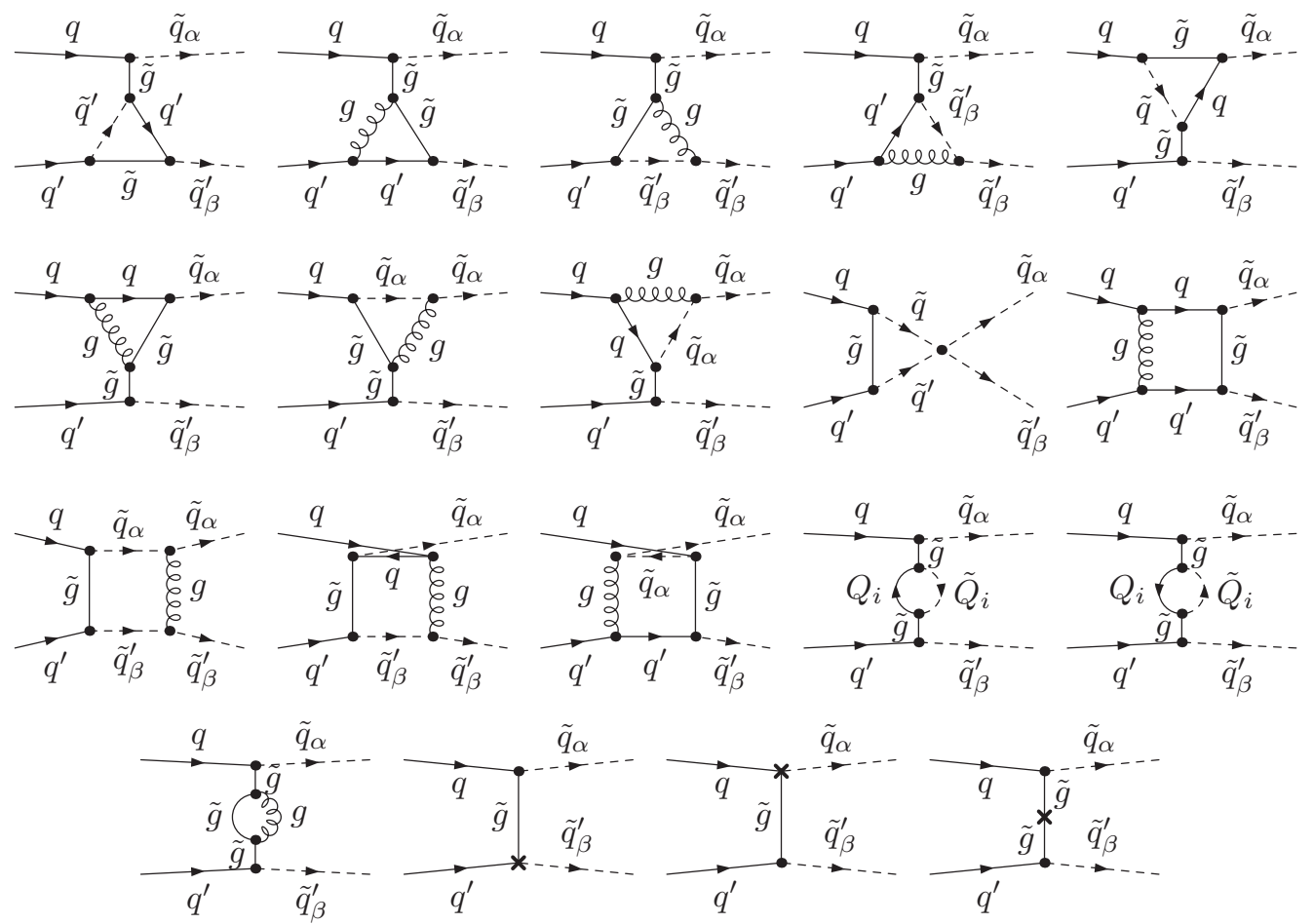

Figure 15. Virtual corrections (III): QCD one-loop insertions into QCD Born diagrams. For $q=q^{\prime}$ crossed diagrams have to be taken into account. Here, $Q_{i}$ can be any of the six quark flavors. The diagram containing the four squark vertex has to be evaluated at $\mathcal{O}\left(\alpha_{s}^{2}\right)$. The renormalization constants appearing in the counter term diagrams (last three diagrams) have to be evaluated at $\mathcal{O}\left(\alpha_{s}\right)$, i.e. the strong sector has to be renormalized.
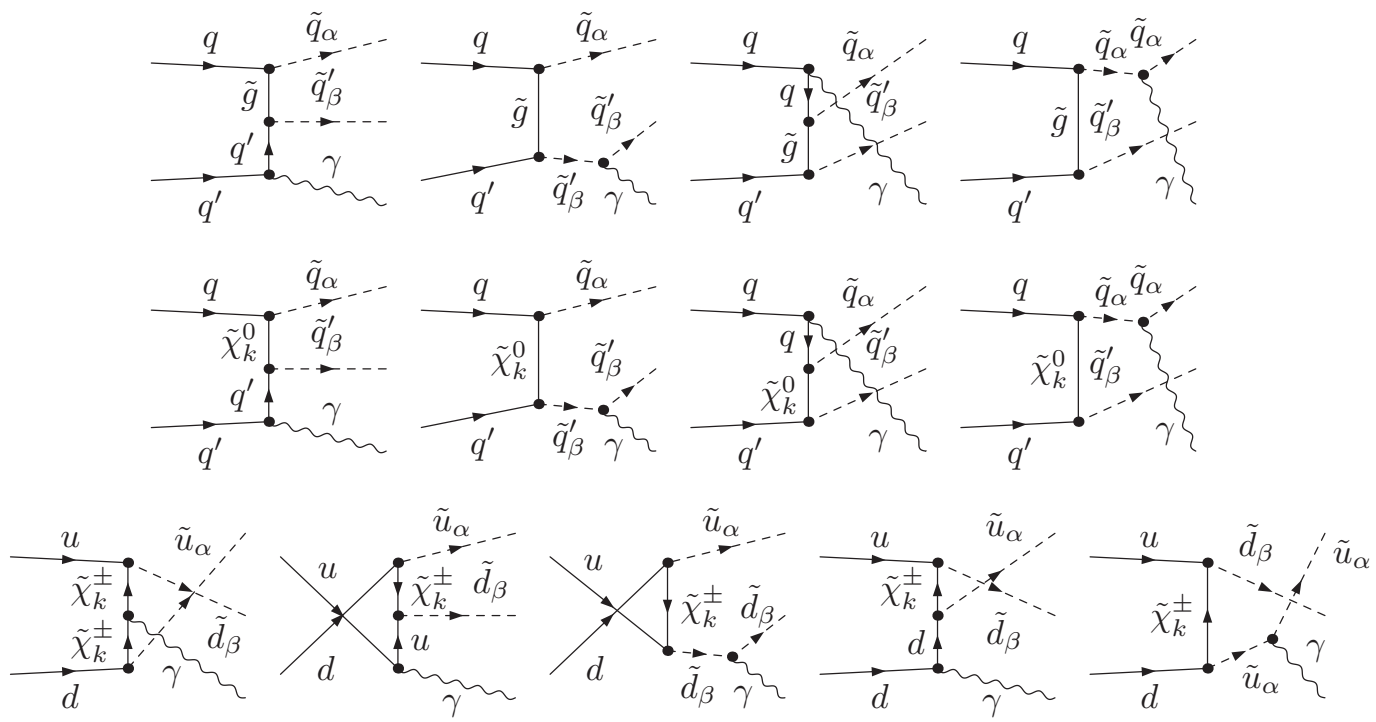

Figure 16. Feynman diagrams for real photon emission. For $q=q^{\prime}$ crossed diagrams have to be taken into account. Diagrams in the last row only contribute for $u=\{u, c\}, d=\{d, s\}$. 

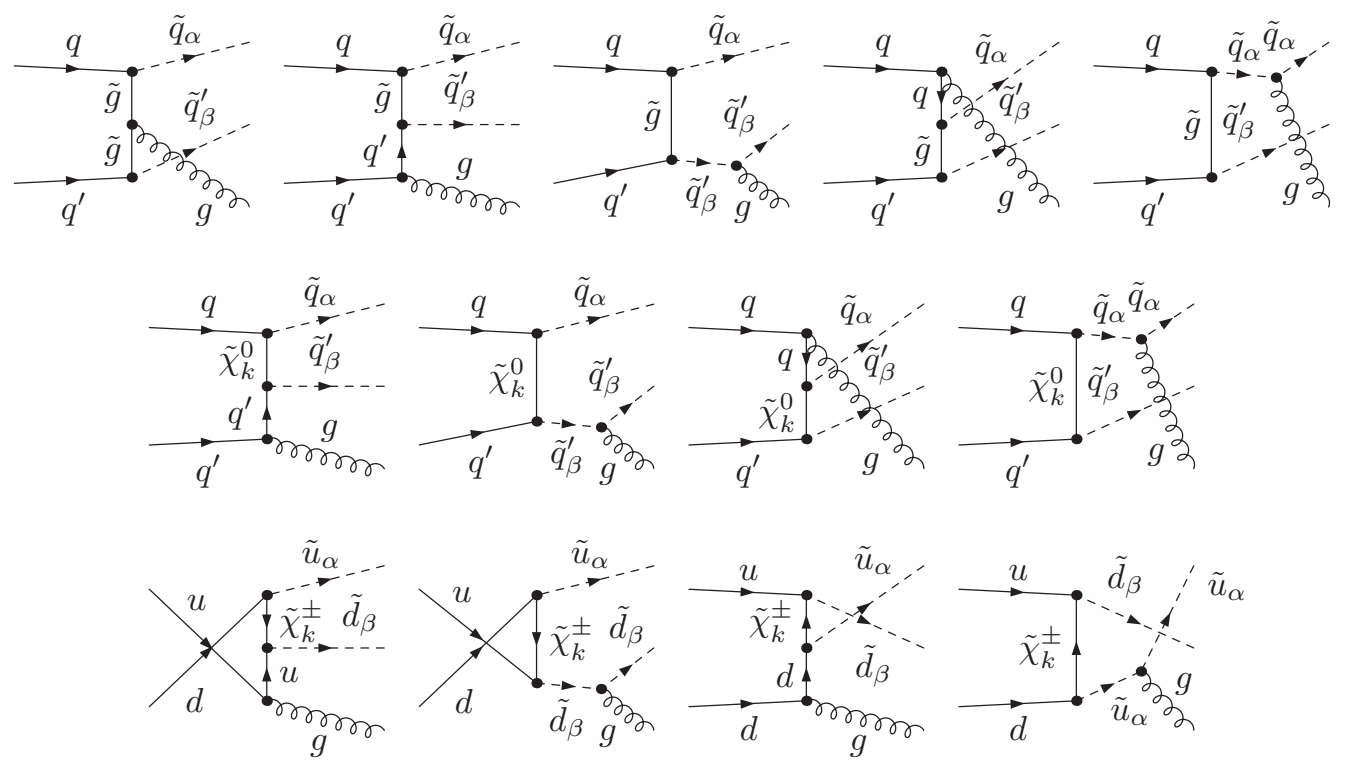

Figure 17. Feynman diagrams for real gluon emission. For $q=q^{\prime}$ crossed diagrams have to be taken into account. Diagrams in the last row only contribute for $u=\{u, c\}, d=\{d, s\}$.
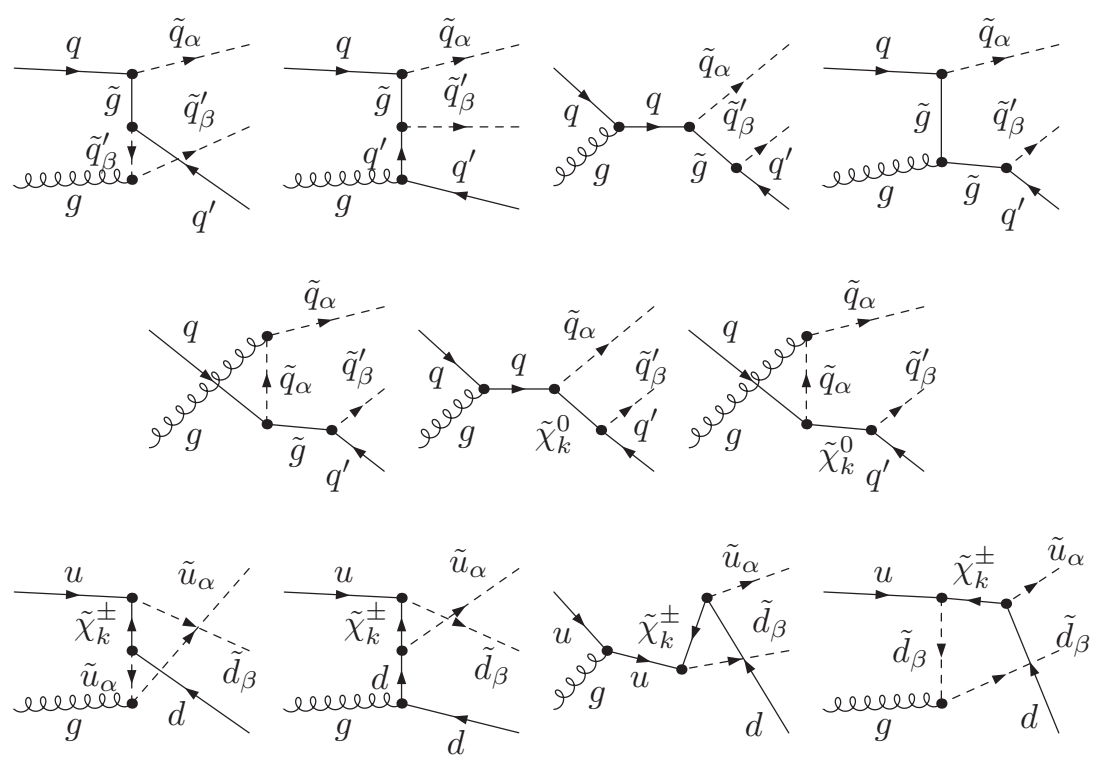

Figure 18. Feynman diagrams for real quark emission. For $q \neq q^{\prime}$ diagrams with $q$ and $q^{\prime}$ exchanged have to be considered, too. For $q=q^{\prime}$ crossed diagrams have to be taken into account. Diagrams in the last row only contribute for $u=\{u, c\}, d=\{d, s\}$.

Open Access. This article is distributed under the terms of the Creative Commons Attribution Noncommercial License which permits any noncommercial use, distribution, and reproduction in any medium, provided the original author(s) and source are credited. 


\section{References}

[1] J. Wess and B. Zumino, Supergauge Transformations in Four-Dimensions, Nucl. Phys. B 70 (1974) 39 [SPIRES].

[2] WMAP collaboration, J. Dunkley et al., Five-Year Wilkinson Microwave Anisotropy Probe (WMAP) Observations: Likelihoods and Parameters from the WMAP data, Astrophys. J. Suppl. 180 (2009) 306 [arXiv:0803.0586] [SPIRES].

[3] H.P. Nilles, Supersymmetry, Supergravity and Particle Physics, Phys. Rept. 110 (1984) 1 [SPIRES].

[4] H.E. Haber and G.L. Kane, The Search for Supersymmetry: Probing Physics Beyond the Standard Model, Phys. Rept. 117 (1985) 75 [SPIRES].

[5] R. Barbieri, Looking Beyond the Standard Model: The Supersymmetric Option, Riv. Nuovo Cim. 11 (1988) 1.

[6] J.R. Ellis, S. Heinemeyer, K.A. Olive, A.M. Weber and G. Weiglein, The Supersymmetric Parameter Space in Light of $B^{-}$physics Observables and Electroweak Precision Data, JHEP 08 (2007) 083 [arXiv:0706.0652] [SPIRES].

[7] O. Buchmueller et al., Prediction for the Lightest Higgs Boson Mass in the CMSSM using Indirect Experimental Constraints, Phys. Lett. B 657 (2007) 87 [arXiv:0707.3447] [SPIRES].

[8] O. Buchmueller et al., Likelihood Functions for Supersymmetric Observables in Frequentist Analyses of the CMSSM and NUHM1, Eur. Phys. J. C 64 (2009) 391 [arXiv:0907.5568] [SPIRES].

[9] Muon G-2 collaboration, G.W. Bennett et al., Measurement of the Positive Muon Anomalous Magnetic Moment to 0.7 ppm, Phys. Rev. Lett. 89 (2002) 101804 [hep-ex/0208001] [SPIRES].

[10] Muon G-2 collaboration, G.W. Bennett et al., Measurement of the negative muon anomalous magnetic moment to 0.7-ppm, Phys. Rev. Lett. 92 (2004) 161802 [hep-ex/0401008] [SPIRES].

[11] O. Buchmueller et al., Predictions for Supersymmetric Particle Masses in the CMSSM using Indirect Experimental and Cosmological Constraints, JHEP 09 (2008) 117 [arXiv:0808.4128] [SPIRES].

[12] CDF collaboration, X. Portell, Searches for squarks and gluinos at CDF and DO detectors, J. Phys. Conf. Ser. 110 (2008) 072035 [hep-ex/0609017] [SPIRES].

[13] P. de Jong, Prospects for SUSY searches in CMS and ATLAS, AIP Conf. Proc. 1078 (2009) 21 [arXiv:0809.3708] [SPIRES].

[14] P.R. Harrison and C.H. Llewellyn Smith, Hadroproduction of Supersymmetric Particles, Nucl. Phys. B 213 (1983) 223 [SPIRES].

[15] E. Reya and D.P. Roy, Supersymmetric particle production at $p \bar{p}$ collider energies, Phys. Rev. D 32 (1985) 645 [SPIRES].

[16] S. Dawson, E. Eichten and C. Quigg, Search for Supersymmetric Particles in Hadron Hadron Collisions, Phys. Rev. D 31 (1985) 1581 [SPIRES].

[17] H. Baer and X. Tata, Component formulae for hadroproduction of left-handed and right-handed squarks, Phys. Lett. B 160 (1985) 159 [SPIRES]. 
[18] W. Beenakker, R. Hopker, M. Spira and P.M. Zerwas, Squark and gluino production at hadron colliders, Nucl. Phys. B 492 (1997) 51 [hep-ph/9610490] [SPIRES].

[19] W. Beenakker, M. Krämer, T. Plehn, M. Spira and P.M. Zerwas, Stop production at hadron colliders, Nucl. Phys. B 515 (1998) 3 [hep-ph/9710451] [SPIRES].

[20] U. Langenfeld and S.-O. Moch, Higher-order soft corrections to squark hadro- production, Phys. Lett. B 675 (2009) 210 [arXiv:0901.0802] [SPIRES].

[21] A. Kulesza and L. Motyka, Threshold resummation for squark-antisquark and gluino- pair production at the LHC, Phys. Rev. Lett. 102 (2009) 111802 [arXiv:0807.2405] [SPIRES].

[22] A. Kulesza and L. Motyka, Soft gluon resummation for the production of gluino-gluino and squark-antisquark pairs at the LHC, Phys. Rev. D 80 (2009) 095004 [arXiv: 0905.4749] [SPIRES].

[23] W. Beenakker et al., Soft-gluon resummation for squark and gluino hadroproduction, JHEP 12 (2009) 041 [arXiv:0909.4418] [SPIRES].

[24] S. Bornhauser, M. Drees, H.K. Dreiner and J.S. Kim, Electroweak Contributions to Squark Pair Production at the LHC, Phys. Rev. D 76 (2007) 095020 [arXiv:0709.2544] [SPIRES].

[25] A. Arhrib, R. Benbrik, K. Cheung and T.-C. Yuan, Higgs boson enhancement effects on squark-pair production at the LHC, JHEP 02 (2010) 048 [arXiv:0911.1820] [SPIRES].

[26] G. Bozzi, B. Fuks, and M. Klasen, Non-diagonal and mixed squark production at hadron colliders, Phys. Rev. D 72 (2005) 035016 [hep-ph/0507073] [SPIRES].

[27] A.T. Alan, K. Cankocak and D.A. Demir, Squark pair production in the MSSM with explicit CP-violation, Phys. Rev. D 75 (2007) 095002 [hep-ph/0702289] [SPIRES].

[28] W. Hollik, M. Kollar and M.K. Trenkel, Hadronic production of top-squark pairs with electroweak NLO contributions, JHEP 02 (2008) 018 [arXiv: 0712.0287] [SPIRES].

[29] W. Hollik and E. Mirabella, Squark anti-squark pair production at the LHC: the electroweak contribution, JHEP 12 (2008) 087 [arXiv:0806.1433] [SPIRES].

[30] W. Hollik, E. Mirabella and M.K. Trenkel, Electroweak contributions to squark-gluino production at the LHC, JHEP 02 (2009) 002 [arXiv:0810.1044] [SPIRES].

[31] M. Beccaria, G. Macorini, L. Panizzi, F.M. Renard and C. Verzegnassi, Stop-antistop and sbottom-antisbottom production at LHC: a one-loop search for model parameters dependence, Int. J. Mod. Phys. A 23 (2008) 4779 [arXiv:0804.1252] [SPIRES].

[32] E. Mirabella, NLO electroweak contributions to gluino pair production at hadron colliders, JHEP 12 (2009) 012 [arXiv:0908.3318] [SPIRES].

[33] T. Hahn, Generating Feynman diagrams and amplitudes with FeynArts 3, Comput. Phys. Commun. 140 (2001) 418 [hep-ph/0012260] [SPIRES].

[34] T. Hahn and C. Schappacher, The implementation of the minimal supersymmetric standard model in FeynArts and FormCalc, Comput. Phys. Commun. 143 (2002) 54 [hep-ph/0105349] [SPIRES].

[35] T. Hahn and M. Rauch, News from FormCalc and LoopTools, Nucl. Phys. Proc. Suppl. 157 (2006) 236 [hep-ph/0601248] [SPIRES].

[36] W. Hollik and D. Stöckinger, Regularization and supersymmetry-restoring counterterms in supersymmetric QCD, Eur. Phys. J. C 20 (2001) 105 [hep-ph/0103009] [SPIRES]. 
[37] U. Baur, S. Keller and D. Wackeroth, Electroweak radiative corrections to $W$ boson production in hadronic collisions, Phys. Rev. D 59 (1999) 013002 [hep-ph/9807417] [SPIRES].

[38] W. Hollik, T. Kasprzik and B.A. Kniehl, Electroweak corrections to W-boson hadroproduction at finite transverse momentum, Nucl. Phys. B 790 (2008) 138 [arXiv:0707.2553] [SPIRES].

[39] LHC/LC Study Group collaboration, G. Weiglein et al., Physics interplay of the LHC and the ILC, Phys. Rept. 426 (2006) 47 [hep-ph/0410364] [SPIRES].

[40] J.A. Aguilar-Saavedra et al., Supersymmetry parameter analysis: SPA convention and project, Eur. Phys. J. C 46 (2006) 43 [hep-ph/0511344] [SPIRES].

[41] Tevatron Electroweak Working Group collaboration, Combination of CDF and DO Results on the Mass of the Top Quark, arXiv:0903.2503 [SPIRES].

[42] B.C. Allanach et al., The Snowmass points and slopes: Benchmarks for SUSY searches, Eur. Phys. J. C 25 (2002) 113 [hep-ph/0202233] [SPIRES].

[43] B.C. Allanach, SOFTSUSY: A C++ program for calculating supersymmetric spectra, Comput. Phys. Commun. 143 (2002) 305 [hep-ph/0104145] [SPIRES].

[44] W. Hollik and H. Rzehak, The sfermion mass spectrum of the MSSM at the one-loop level, Eur. Phys. J. C 32 (2003) 127 [hep-ph/0305328] [SPIRES].

[45] A.D. Martin, R.G. Roberts, W.J. Stirling and R.S. Thorne, Parton distributions incorporating QED contributions, Eur. Phys. J. C 39 (2005) 155 [hep-ph/0411040] [SPIRES].

[46] S. Dittmaier and M. Krämer, 1, Electroweak radiative corrections to $W$-boson production at hadron colliders, Phys. Rev. D 65 (2002) 073007 [hep-ph/0109062] [SPIRES].

[47] A.D. Martin, R.G. Roberts, W.J. Stirling and R.S. Thorne, Physical gluons and high E(T) jets, Phys. Lett. B 604 (2004) 61 [hep-ph/0410230] [SPIRES].

[48] A. Denner, Techniques for calculation of electroweak radiative corrections at the one loop level and results for W physics at LEP-200, Fortschr. Phys. 41 (1993) 307 [arXiv: 0709.1075] [SPIRES].

[49] S. Catani and M.H. Seymour, A general algorithm for calculating jet cross sections in NLO QCD, Nucl. Phys. B 485 (1997) 291 [hep-ph/9605323] [SPIRES]. 